\title{
Synergistic Effect between Cryptotanshinone and Antibiotics against Clinic Methicillin and Vancomycin-Resistant Staphylococcus aureus
}

\author{
Jeong-Dan Cha, ${ }^{1}$ Jeong-Ho Lee, ${ }^{2}$ Kyung Min Choi, ${ }^{1}$ Sung-Mi Choi, ${ }^{3}$ and Jeong Hye Park ${ }^{4}$ \\ ${ }^{1}$ Department of Research Development, Institute of Jinan Red Ginseng, Jinan, Republic of Korea \\ ${ }^{2}$ Department of Oriental Medicine Resources, College of Environmental and Bioresource Sciences, \\ Chonbuk National University, Republic of Korea \\ ${ }^{3}$ Department of Dental Hygiene, Daegu Health College, Daegu, Republic of Korea \\ ${ }^{4}$ Department of Nursing, Dong-eui University, Republic of Korea
}

Correspondence should be addressed to Jeong Hye Park; jhpark@deu.ac.kr

Received 25 November 2013; Revised 6 February 2014; Accepted 18 February 2014; Published 24 March 2014

Academic Editor: Tai-Long Pan

Copyright (C) 2014 Jeong-Dan Cha et al. This is an open access article distributed under the Creative Commons Attribution License, which permits unrestricted use, distribution, and reproduction in any medium, provided the original work is properly cited.

Cryptotanshinone (CT), a major tanshinone of medicinal plant Salvia miltiorrhiza Bunge, demonstrated strong antibacterial activity against clinic isolated methicillin and vancomycin-resistant Staphylococcus aureus (MRSA and VRSA) in this experiment. The CT was determined against clinic isolated MRSA 1-16 with MIC and MBC values ranging from 4 to 32 and 8 to $128 \mu \mathrm{g} / \mathrm{mL}$; for MSSA 1-2 from 16 to $32 \mu \mathrm{g} / \mathrm{mL}$ and 64 to $128 \mu \mathrm{g} / \mathrm{mL}$; for VRSA $1-2$ from 2 to $4 \mu \mathrm{g} / \mathrm{mL}$ and 4 to $16 \mu \mathrm{g} / \mathrm{mL}$, respectively. The range of $\mathrm{MIC}_{50}$ and $\mathrm{MIC}_{90}$ of CT was $0.5-8 \mu \mathrm{g} / \mathrm{mL}$ and $4-64 \mu \mathrm{g} / \mathrm{mL}$, respectively. The combination effects of CT with antibiotics were synergistic (FIC index < 0.5) against most of tested clinic isolated MRSA, MSSA, and VRSA except additive, MRSA 4 and 16 in oxacillin, MRSA 6, 12, and 15 in ampicillin, and MRSA 6, 11, and 15 in vancomycin (FIC index < 0.75-1.0). Furthermore, a time-kill study showed that the growth of the tested bacteria was completely attenuated after 2-6h of treatment with the 1/2 MIC of CT, regardless of whether it was administered alone or with ampicillin, oxacillin, or vancomycin. The results suggest that CT could be employed as a natural antibacterial agent against multidrug-resistant pathogens infection.

\section{Introduction}

Staphylococcus aureus (S. aureus) is one of the most important pathogens in both hospitals and the community and can cause numerous syndromes in humans, such as furuncle, carbuncle, abscess, pneumonia, meningitis, bacterial arthritis, myocarditis, endocarditis, osteomyelitis, and septicemia [13]. Methicillin-resistant Staphylococcus aureus (MRSA) is a significant problem in hospitals and communities worldwide, and awareness of MRSA in animals and reports of its zoonotic spread have increased in recent years $[3,4]$. The increasing prevalence of methicillin-resistant Staphylococcus aureus (MRSA) has led to widespread, increased use of vancomycin $[5,6]$. Subsequently, numerous reports of elevated minimum inhibitory concentrations (MIC) to vancomycin among MRSA isolates have surfaced, concomitant with increased global vancomycin exposure to MRSA [7, 8]. Greater than $60 \%$ of $S$. aureus isolates are now resistant to methicillin (oxacillin), and some strains have developed resistance to more than 20 different antimicrobial agents; new agents are therefore needed for the treatment of $S$. aureus $[9,10]$. Plant medicines are used on a worldwide scale to prevent and treat infectious diseases. They are of great demand both in the developed and developing countries for the primary health care needs due to their wide biological and medicinal activities, higher safety margin and lesser costs $[11,12]$. Plants are rich in a wide variety of secondary metabolites such as tannins, alkaloids, terpenoids, and flavonoids having been found in vitro since they have antimicrobial properties and may serve as an alternative, effective, cheap, and safe antimicrobial for the treatment of microbial infections [12-15]. At the same time, 
TABLE 1: Antibacterial activity of cryptotanshinone and antibiotics in isolated MRSA, VRSA, MSSA, and some of reference bacteria.

\begin{tabular}{|c|c|c|c|c|c|c|}
\hline \multirow{2}{*}{ Samples } & \multicolumn{3}{|c|}{ Cryptotanshinone $(\mu \mathrm{g} / \mathrm{mL})$} & \multirow[t]{2}{*}{ Ampicillin } & \multirow{2}{*}{$\begin{array}{c}\text { Oxacillin } \\
\text { MIC/MBC }(\mu \mathrm{g} / \mathrm{mL})\end{array}$} & \multirow[t]{2}{*}{ Vancomycin } \\
\hline & $\mathrm{MIC}_{50<}$ & $\mathrm{MIC}_{90<}$ & $\mathrm{MIC} / \mathrm{MBC}$ & & & \\
\hline MSSA ATCC $25923^{1}$ & 4 & 64 & $64 / 256$ & $8 / 16$ & $0.25 / 1$ & $0.5 / 2$ \\
\hline MRSA ATCC $33591^{2}$ & 0.5 & 4 & $4 / 16$ & $512 / 2048$ & $8 / 16$ & $1 / 4$ \\
\hline VRSA $3 A 063^{3}$ & 0.5 & 2 & $2 / 4$ & $512 / 2048$ & $512 / 1024$ & $16 / 32$ \\
\hline VRSA $3 A 066^{4}$ & 1 & 4 & $4 / 16$ & $128 / 256$ & $512 / 1024$ & $32 / 64$ \\
\hline MSSA $1^{5}$ & 2 & 16 & $16 / 64$ & $1024 / 2048$ & $0.5 / 1$ & $1 / 4$ \\
\hline $\operatorname{MSSA} 2^{6}$ & 8 & 32 & $32 / 128$ & $256 / 512$ & $1 / 2$ & $1 / 2$ \\
\hline MRSA 1 & 2 & 16 & $16 / 32$ & $128 / 256$ & $128 / 256$ & $1 / 2$ \\
\hline MRSA 2 & 8 & 64 & $64 / 128$ & $256 / 256$ & $8 / 32$ & $2 / 4$ \\
\hline MRSA 3 & 1 & 4 & $4 / 16$ & $128 / 512$ & $128 / 512$ & $2 / 4$ \\
\hline MRSA 4 & 0.5 & 4 & $4 / 8$ & $128 / 256$ & $512 / 2048$ & $1 / 2$ \\
\hline MRSA 5 & 2 & 64 & $64 / 128$ & $128 / 512$ & $512 / 1024$ & $1 / 2$ \\
\hline MRSA 6 & 0.5 & 4 & $4 / 16$ & $64 / 128$ & $64 / 256$ & $1 / 2$ \\
\hline MRSA 7 & 2 & 8 & $8 / 16$ & $128 / 256$ & $512 / 1024$ & $1 / 4$ \\
\hline MRSA 8 & 4 & 32 & $32 / 64$ & $256 / 256$ & $512 / 2048$ & $1 / 4$ \\
\hline MRSA 9 & 2 & 8 & $8 / 16$ & $64 / 128$ & $512 / 1024$ & $1 / 2$ \\
\hline MRSA 10 & 2 & 16 & $16 / 64$ & $128 / 256$ & $512 / 1024$ & $1 / 2$ \\
\hline MRSA 11 & 4 & 8 & $8 / 32$ & $64 / 128$ & $64 / 128$ & $1 / 2$ \\
\hline MRSA 12 & 1 & 4 & $4 / 8$ & $128 / 256$ & $128 / 256$ & $1 / 4$ \\
\hline MRSA 13 & 4 & 32 & $32 / 128$ & $128 / 256$ & $32 / 128$ & $1 / 2$ \\
\hline MRSA 14 & 0.5 & 8 & $8 / 16$ & $64 / 128$ & $8 / 16$ & $1 / 4$ \\
\hline MRSA 15 & 0.5 & 4 & $4 / 16$ & $64 / 128$ & $128 / 256$ & $1 / 4$ \\
\hline MRSA 16 & 2 & 8 & $8 / 16$ & $128 / 512$ & $128 / 512$ & $1 / 2$ \\
\hline
\end{tabular}

${ }^{1}$ MSSA (ATCC 25923): reference strain methicillin-sensitive Staphylococcus aureus.

${ }^{2}$ MRSA (ATCC 33591): reference strain methicillin-resistant Staphylococcus aureus.

${ }^{3}$ VRSA 3A063: vancomycin-resistant Staphylococcus aureus isolated clinically.

${ }^{4}$ VRSA 3A066: vancomycin-resistant Staphylococcus aureus isolated clinically.

${ }^{5}$ MSSA (1-2): methicillin-sensitive Staphylococcus aureus isolated clinically.

${ }^{6}$ MRSA (1-16): methicillin-resistant Staphylococcus aureus isolated clinically.

because of the difficulty in developing chemical synthetic drugs and because of their side effects, scientists are making more efforts to search for new drugs from plant resources to combat clinical multidrug-resistant microbial infections $[11,12,16]$.

The main components of $S$. miltiorrhiza can be divided into two groups: hydrophilic compounds such as salvianolic acids and lipophilic chemicals, including diterpenoid and tanshinones [17]. The second group of components, labeled as tanshinone I, tanshinone II, and cryptotanshinone, are the major bioactive constituents and have various kinds of pharmacological effects including antibacterial, antioxidant, and antitumor activities and prevention of angina pectoris and myocardial infarction [18-20]. The cryptotanshinone (CT) exhibits antimicrobial activity against a broad range of Gram-positive bacteria, including S. aureus, and Gramnegative bacteria as well as other microorganisms $[18,21]$. The hexane and chloroform fractions of S. miltiorrhiza evidenced profound antimicrobial activity and inhibited resistant gene expression against Staphylococcus aureus and MRSA (methicillin-resistant Staphylococcus aureus) [21, 22].

In this study, the antimicrobial activities of cryptotanshinone (CT) against methicillin and vancomycin-resistant
Staphylococcus aureus isolated in a clinic were assessed using broth microdilution method and the checkerboard and timekill methods for synergistic effect of the combination with antibiotics.

\section{Materials and Methods}

2.1. Plant Extraction and Isolation. The air-dried roots $(2.0 \mathrm{~kg})$ of $S$. miltiorrhiza were crushed and extracted with $\mathrm{MeOH}(8 \mathrm{~L} \times 3)$ at room temperature. The $\mathrm{MeOH}$ extracts $(210 \mathrm{~g})$ were evaporated and suspended in distilled water and partitioned sequentially with $\mathrm{CH}_{2} \mathrm{Cl}_{2}$, EtOAc, and n$\mathrm{BuOH}$. The $\mathrm{CH}_{2} \mathrm{Cl}_{2}$ soluble fraction was concentrated in vacuo, and its crude extract $(28 \mathrm{~g})$ was subjected to silica gel column chromatography using a $\mathrm{CHCl}_{3}-\mathrm{MeOH}$ gradient solvent system to provide ten fractions (A1-A10). Fraction A4 was further subjected to silica gel column chromatography with a $\mathrm{CHCl}_{3}-\mathrm{MeOH}(10: 1)$ to yield five fractions (B1-B5). Fraction B3 was purified on recycling prep-HPLC (JAIGEL GS column and $220 \mathrm{~nm}$ ) eluted with $\mathrm{MeOH}(4.0 \mathrm{~mL} / \mathrm{min})$ to yield cryptotanshinone $(98 \mathrm{mg})$. The structure of cryptotanshinone was identified by comparing its spectral data with those published $[23,24]$. 

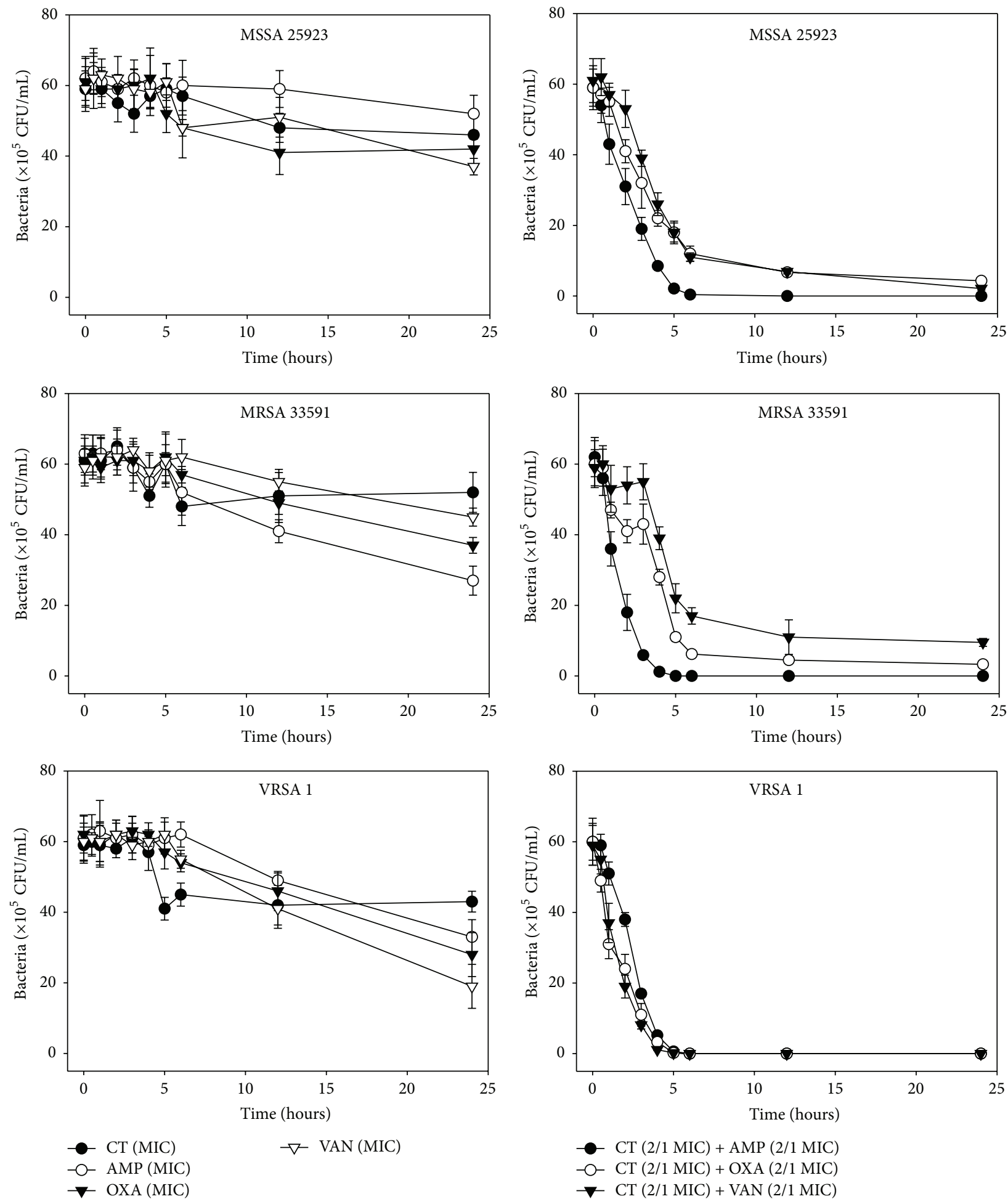

FIgURE 1: Time-kill curves of MIC or 1/2 MIC of cryptotanshinone (CT), ampicillin (AMP), oxacillin (OXA), and vancomycin (VAN) alone and its combination with $\mathrm{MIC}_{50}$ of AMP or OXA, and VAN against VRSA 1 isolates and reference stains, MSSA ATCC 25923 and MRSA ATCC 33591. Bacteria were incubated with MIC of CT (•), AMP, OXA, and VAN, and 1/2 MIC of CT + 1/2 MIC of AMP (O), 1/2 MIC of CT $+1 / 2$ MIC of OXA $(\boldsymbol{\nabla})$, and 1/2 MIC of CT + 1/2 MIC of VAN $(\nabla)$ over time. CFU: colony-forming units. 

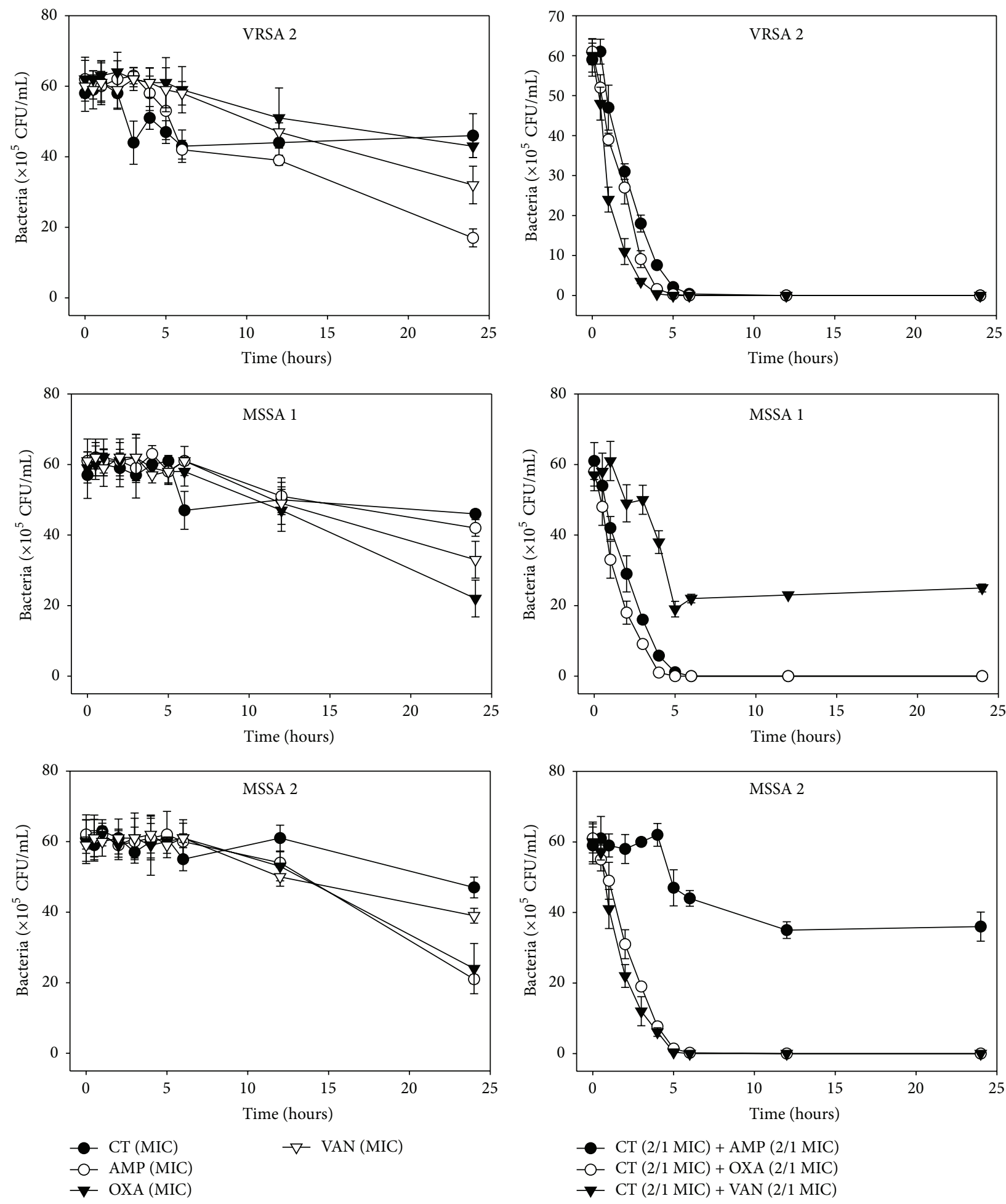

FIgURE 2: Time-kill curves of MIC or 1/2 MIC of cryptotanshinone (CT), ampicillin (AMP), oxacillin (OXA), and vancomycin (VAN) alone and its combination with 1/2 MIC of AMP or OXA, and VAN against VRSA 2, MSSA 1, and MSSA 2 isolates. Bacteria were incubated with MIC of CT ( ), AMP, OXA, and VAN, and 1/2 MIC of CT + 1/2 MIC of AMP (O), 1/2 MIC of CT + 1/2 MIC of OXA ( $\boldsymbol{\nabla})$, and 1/2 MIC of CT $+1 / 2$ MIC of VAN $(\nabla)$ over time. CFU: colony-forming units. 

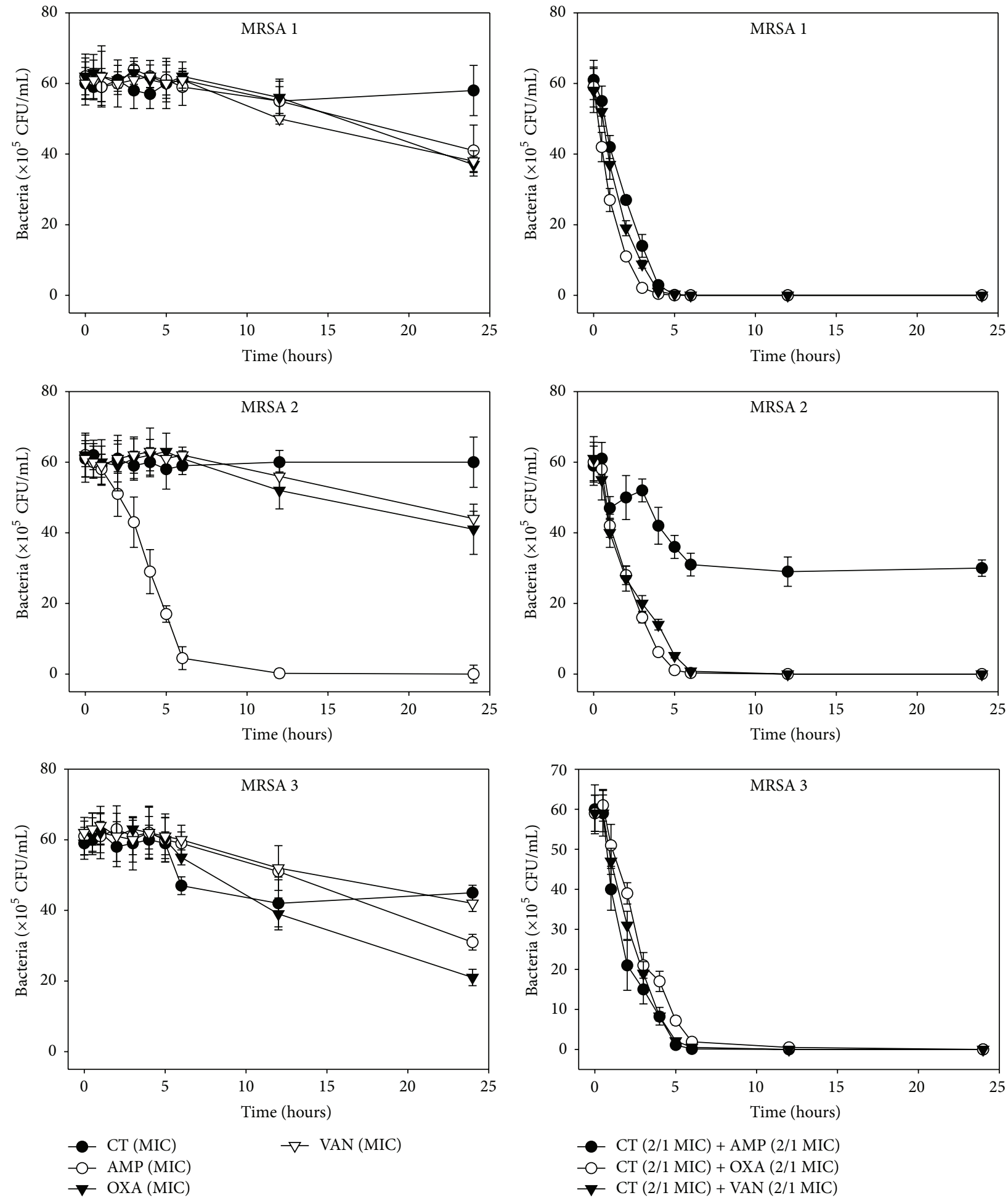

FIGURE 3: Time-kill curves of MIC or 1/2 MIC of cryptotanshinone (CT), ampicillin (AMP), oxacillin (OXA), and vancomycin (VAN) alone and its combination with 1/2 MIC of AMP or OXA, and VAN against MRSA 1, 2, and 3 isolates. Bacteria were incubated with MIC of CT (•), AMP, OXA, and VAN, and 1/2 MIC of CT + 1/2 MIC of AMP (O), 1/2 MIC of CT + 1/2 MIC of OXA ( $\nabla)$, and 1/2 MIC of CT + 1/2 MIC of VAN $(\nabla)$ over time. CFU: colony-forming units. 

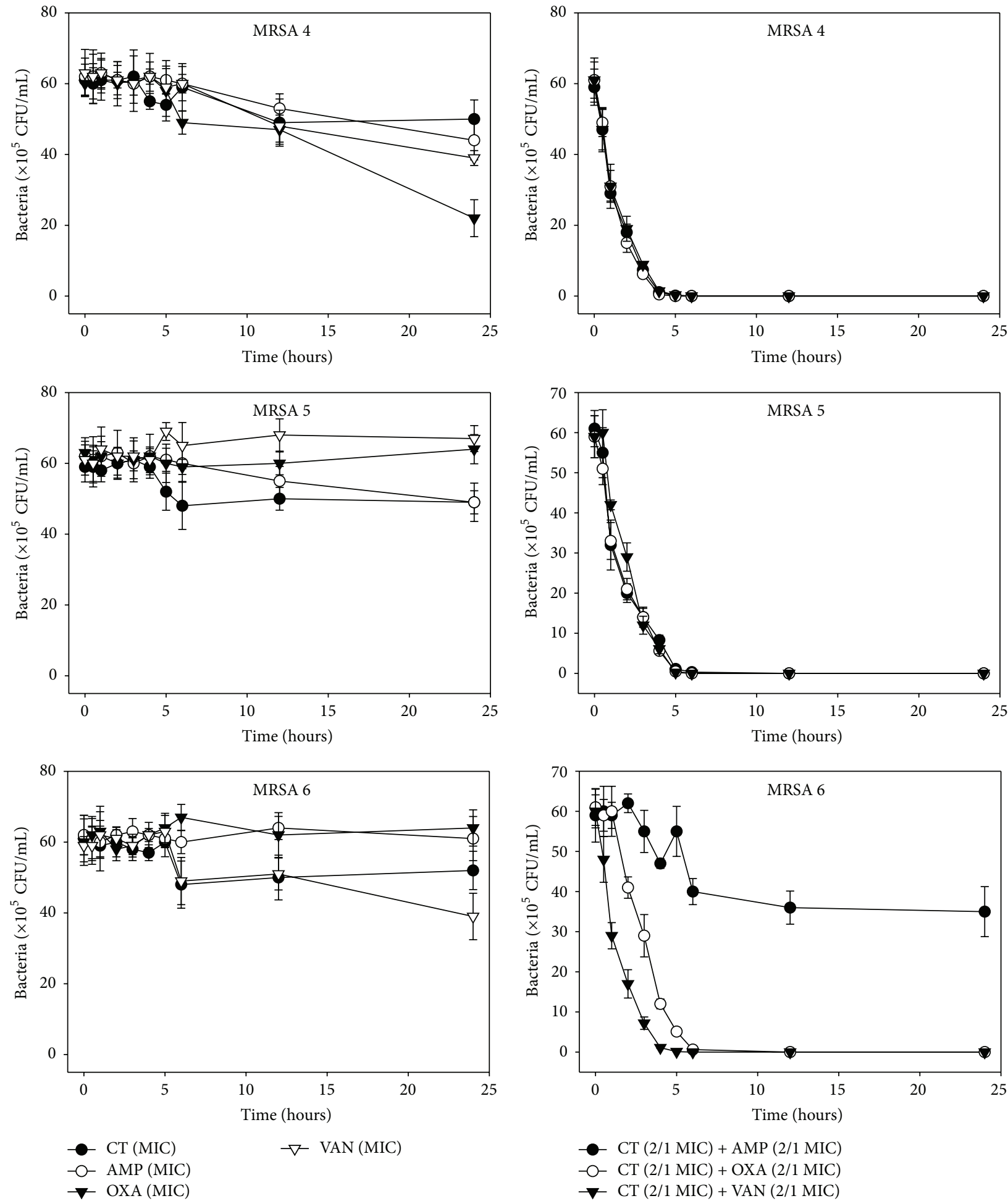

FIGURE 4: Time-kill curves of MIC or 1/2 MIC of cryptotanshinone (CT), ampicillin (AMP), oxacillin (OXA), and vancomycin (VAN) alone and its combination with 1/2 MIC of AMP or OXA, and VAN against MRSA 4, 5, and 6 isolates. Bacteria were incubated with MIC of CT $(\bullet)$, AMP $(O)$, OXA $(\boldsymbol{\nabla})$, and VAN $(\nabla)$, and 1/2 MIC of CT + 1/2 MIC of AMP (•), 1/2 MIC of CT + 1/2 MIC of OXA (O), and 1/2 MIC of CT $+1 / 2$ MIC of VAN $(\boldsymbol{\nabla})$ over time. CFU: colony-forming units. 

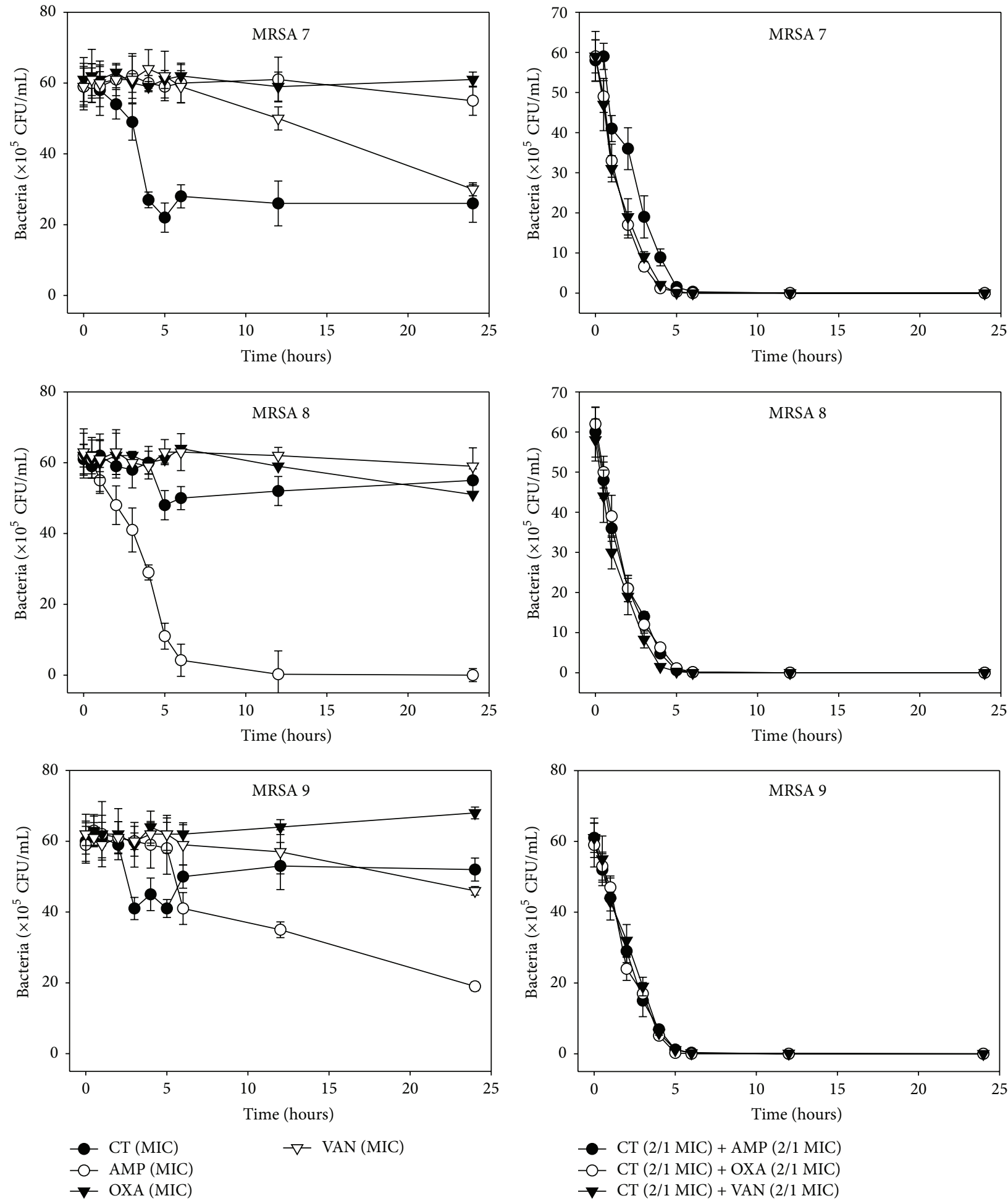

FIGURE 5: Time-kill curves of MIC or 1/2 MIC of cryptotanshinone (CT), ampicillin (AMP), oxacillin (OXA), and vancomycin (VAN) alone and its combination with 1/2 MIC of AMP or OXA, and VAN against MRSA 7, 8, and 9 isolates. Bacteria were incubated with MIC of CT (•),

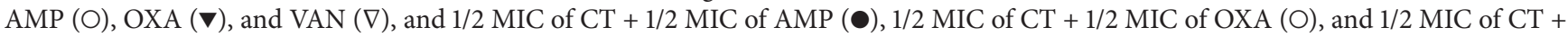
1/2 MIC of VAN $(\boldsymbol{\nabla})$ over time. CFU: colony-forming units. 

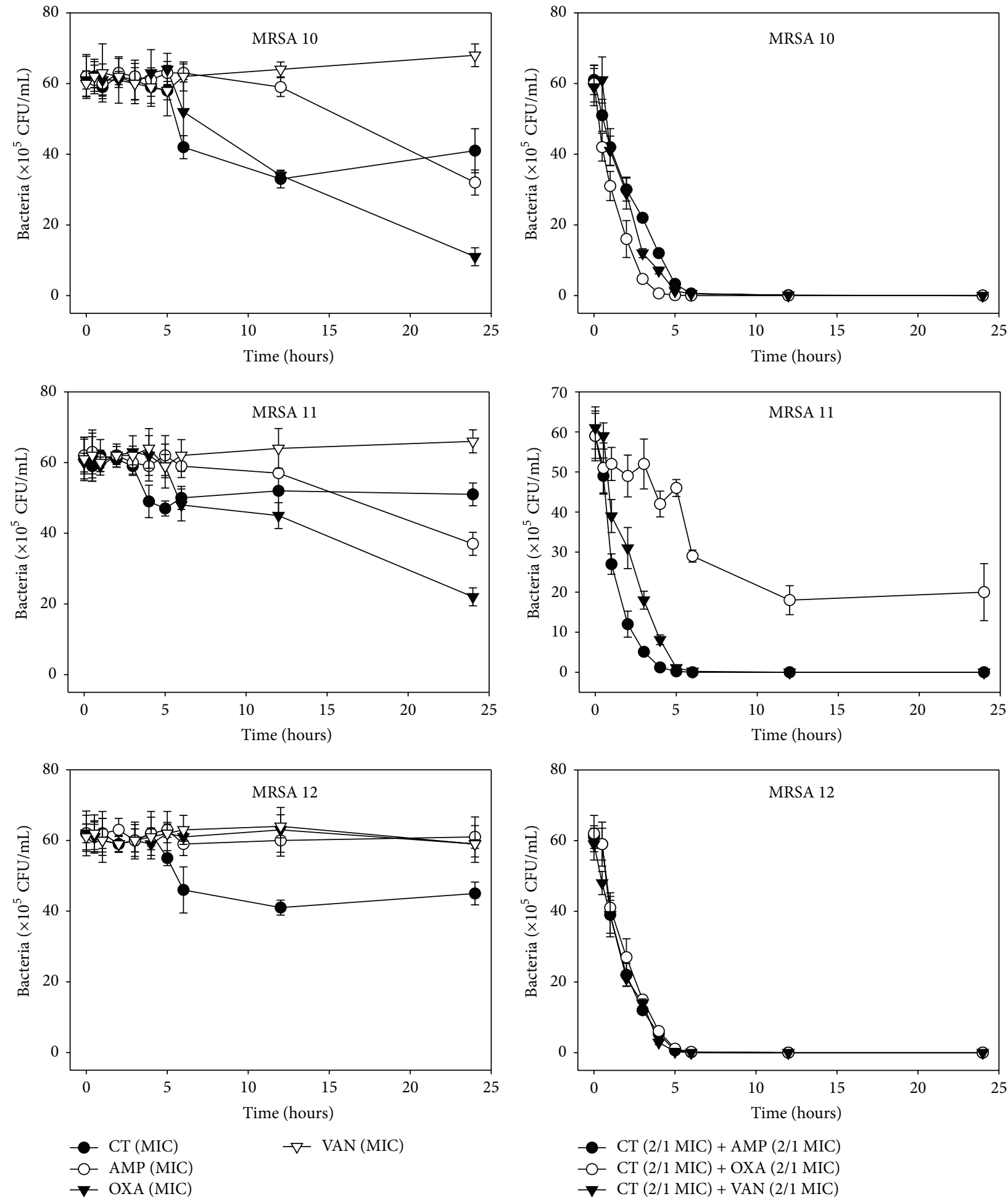

FIGURE 6: Time-kill curves of MIC or 1/2 MIC of cryptotanshinone (CT), ampicillin (AMP), oxacillin (OXA), and vancomycin (VAN) alone and its combination with 1/2 MIC of AMP or OXA, and VAN against MRSA 10, 11, and 12 isolates. Bacteria were incubated with MIC of CT $(\bullet)$, AMP $(O)$, OXA $(\nabla)$, and VAN $(\nabla)$, and 1/2 MIC of CT + 1/2 MIC of AMP (•), 1/2 MIC of CT + 1/2 MIC of OXA (O), and 1/2 MIC of CT $+1 / 2$ MIC of VAN $(\boldsymbol{\nabla})$ over time. CFU: colony-forming units. 

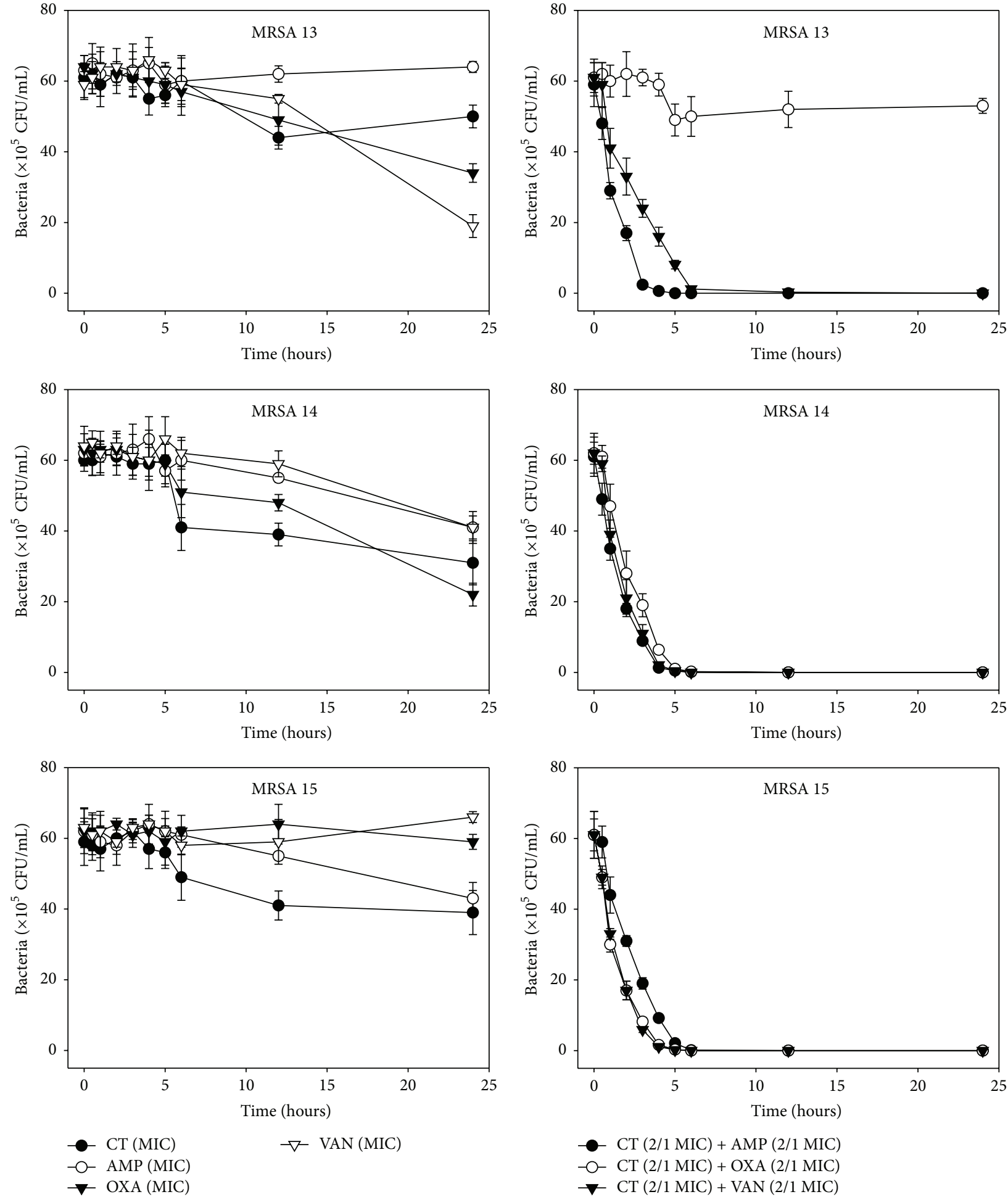

FIGURE 7: Time-kill curves of MIC or 1/2 MIC of cryptotanshinone (CT), ampicillin (AMP), oxacillin (OXA), and vancomycin (VAN) alone and its combination with 1/2 MIC of AMP or OXA, and VAN against MRSA 13, 14, and 15 isolates. Bacteria were incubated with MIC of CT $(\bullet)$, AMP $(O)$, OXA $(\nabla)$, and VAN $(\nabla)$, and 1/2 MIC of CT + 1/2 MIC of AMP (•), 1/2 MIC of CT + 1/2 MIC of OXA (O), and 1/2 MIC of CT $+1 / 2$ MIC of VAN $(\boldsymbol{\nabla})$ over time. CFU: colony-forming units. 
TABLE 2: Synergistic effects of the cryptotanshinone with oxacillin in isolated MRSA, VRSA, MSSA, and some of reference bacteria.

\begin{tabular}{|c|c|c|c|c|c|c|}
\hline \multirow{2}{*}{ Samples } & \multirow{2}{*}{ Agent } & \multicolumn{2}{|c|}{$\mathrm{MIC} / \mathrm{MBC}(\mu \mathrm{g} / \mathrm{mL})$} & \multirow{2}{*}{$\mathrm{FIC} / \mathrm{FBC}$} & \multirow{2}{*}{$\mathrm{FICI} / \mathrm{FBCI}^{2}$} & \multirow{2}{*}{ Outcome } \\
\hline & & Alone & Combination $^{1}$ & & & \\
\hline \multirow{2}{*}{ MSSA ATCC $25923^{3}$} & Cryptotanshinone & $64 / 256$ & $8 / 32$ & $0.125 / 0.125$ & \multirow{2}{*}{$0.375 / 0.375$} & \multirow{2}{*}{ Synergistic/synergistic } \\
\hline & Oxacillin & $0.25 / 1$ & $0.0625 / 0.25$ & $0.25 / 0.25$ & & \\
\hline \multirow{2}{*}{ MRSA ATCC $33591^{4}$} & Cryptotanshinone & $4 / 16$ & $1 / 4$ & $0.25 / 0.25$ & \multirow{2}{*}{$0.5 / 0.5$} & \multirow{2}{*}{ Synergistic/synergistic } \\
\hline & Oxacillin & $8 / 16$ & $2 / 4$ & $0.25 / 0.25$ & & \\
\hline \multirow{2}{*}{ VRSA $3 \mathrm{~A} 063^{5}$} & Cryptotanshinone & $2 / 4$ & $0.5 / 2$ & $0.25 / 0.5$ & \multirow{2}{*}{$0.375 / 0.375$} & \multirow{2}{*}{ Synergistic/synergistic } \\
\hline & Oxacillin & $512 / 1024$ & $64 / 128$ & $0.125 / 0.125$ & & \\
\hline \multirow{2}{*}{ VRSA 3 A $066^{6}$} & Cryptotanshinone & $4 / 16$ & $1 / 4$ & $0.25 / 0.25$ & \multirow{2}{*}{$0.375 / 0.5$} & \multirow{2}{*}{ Synergistic/synergistic } \\
\hline & Oxacillin & $512 / 1024$ & $64 / 256$ & $0.125 / 0.25$ & & \\
\hline \multirow{2}{*}{ MSSA $1^{7}$} & Cryptotanshinone & $16 / 64$ & $2 / 8$ & $0.125 / 0.125$ & \multirow{2}{*}{$0.375 / 0.25$} & \multirow{2}{*}{ Synergistic/synergistic } \\
\hline & Oxacillin & $0.5 / 1$ & $0.125 / 0.125$ & $0.25 / 0.125$ & & \\
\hline \multirow{2}{*}{ MSSA 2} & Cryptotanshinone & $32 / 128$ & $16 / 32$ & $0.5 / 0.25$ & \multirow{2}{*}{$0.375 / 0.375$} & Synergistic/synergistic \\
\hline & Oxacillin & $1 / 2$ & $0.125 / 0.25$ & $0.125 / 0.125$ & & \\
\hline MRSA $1^{8}$ & Cryptotanshinone & $16 / 32$ & $4 / 16$ & $0.25 / 0.5$ & $0.5 / 0.75$ & Synergistic/additive \\
\hline VIKSA I & Oxacillin & $128 / 256$ & $32 / 64$ & $0.25 / 0.25$ & $0.0 / 0.13$ & 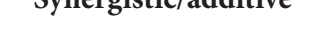 \\
\hline & Cryptotanshinone & $64 / 128$ & $16 / 16$ & $0.25 / 0.125$ & & Synergistic/synergistic \\
\hline MRSA 2 & Oxacillin & $8 / 32$ & $2 / 8$ & $0.25 / 0.25$ & $0.5 / 0.3 / 5$ & synergistic/synergistic \\
\hline MRSA 3 & Cryptotanshinone & $4 / 16$ & $0.5 / 4$ & $0.125 / 0.25$ & $0.375 / 0.375$ & Synergistic/synergistic \\
\hline 10110 & Oxacillin & $128 / 512$ & $32 / 64$ & $0.25 / 0.125$ & (2) & उулет \\
\hline MRSA 4 & Cryptotanshinone & $4 / 8$ & $2 / 2$ & $0.5 / 0.25$ & $0.75 / 0.375$ & Additive/syneroistic \\
\hline (1) & Oxacillin & $512 / 2048$ & $128 / 256$ & $0.25 / 0.125$ & (2) & 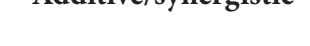 \\
\hline MRSA 5 & Cryptotanshinone & $64 / 128$ & $16 / 32$ & $0.25 / 0.25$ & $0.375 / 0.5$ & Synergistic/synergistic \\
\hline NIKSA 5 & Oxacillin & $512 / 1024$ & $64 / 256$ & $0.125 / 0.25$ & & \\
\hline MRSA 6 & Cryptotanshinone & $4 / 16$ & $1 / 4$ & $0.25 / 0.25$ & $0.5 / 0.5$ & Synergistic/synergistic \\
\hline IVRSA O & Oxacillin & $64 / 256$ & $16 / 64$ & $0.25 / 0.25$ & $0.5 / 0.5$ & synergistic/synergistic \\
\hline MRSA 7 & Cryptotanshinone & $8 / 16$ & $2 / 8$ & $0.25 / 0.5$ & $0.375 / 0.75$ & Synergistic/additive \\
\hline & Oxacillin & $512 / 1024$ & $64 / 256$ & $0.125 / 0.25$ & & \\
\hline MRSA 8 & Cryptotanshinone & $32 / 64$ & $8 / 32$ & $0.25 / 0.5$ & $0.5 / 0.625$ & Synergistic/synergistic \\
\hline VINSA O & Oxacillin & $512 / 2048$ & $128 / 256$ & $0.25 / 0.125$ & $0.5 / 0.025$ & 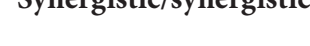 \\
\hline MRSA 9 & Cryptotanshinone & $8 / 16$ & $2 / 4$ & $0.25 / 0.25$ & $0.375 / 0.3125$ & Synergistic/synergistic \\
\hline MIRSA Y & Oxacillin & $512 / 1024$ & $64 / 64$ & $0.125 / 0.0625$ & $0.5 / 2 / 0.5125$ & synergistic/synergistic \\
\hline MRSA 10 & Cryptotanshinone & $16 / 64$ & $2 / 8$ & $0.125 / 0.125$ & $0.3125 / 0.25$ & Synergistic/synergistic \\
\hline IVINUת IO & Oxacillin & $512 / 1024$ & $32 / 128$ & $0.0625 / 0.125$ & & \\
\hline MRSA 11 & Cryptotanshinone & $8 / 32$ & $1 / 4$ & $0.125 / 0.125$ & $0.375 / 0.375$ & Synergistic/synergistic \\
\hline NINOA II & Oxacillin & $64 / 128$ & $16 / 64$ & $0.25 / 0.5$ & $0.5 / 2 / 0.5 / 2$ & synergisto/syiergistic \\
\hline MRSA 12 & Cryptotanshinone & $4 / 8$ & $1 / 2$ & $0.25 / 0.25$ & $0.5 / 0.5$ & Synergistic/synergistic \\
\hline IVIKSA 12 & Oxacillin & $128 / 256$ & $32 / 64$ & $0.25 / 0.25$ & $0.5 / 0.3$ & \\
\hline MRSA 13 & Cryptotanshinone & $32 / 128$ & $8 / 32$ & $0.25 / 0.25$ & $0.5 / 0.375$ & Synergistic/synergistic \\
\hline IVIKSA IJ & Oxacillin & $32 / 128$ & $8 / 8$ & $0.25 / 0.125$ & 0.010 .572 & 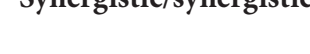 \\
\hline MRSA 14 & Cryptotanshinone & $8 / 16$ & $2 / 4$ & $0.25 / 0.25$ & $0.375 / 0.5$ & Synergistic/synergistic \\
\hline & Oxacillin & $8 / 16$ & $1 / 4$ & $0.125 / 0.25$ & 0.07010 .0 & 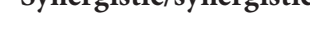 \\
\hline MRSA 15 & Cryptotanshinone & $4 / 16$ & $1 / 8$ & $0.25 / 0.5$ & $0.5 / 0.75$ & Synergistic/additive \\
\hline & Oxacillin & $128 / 256$ & $32 / 64$ & $0.25 / 0.25$ & & \\
\hline & Cryptotanshinone & $8 / 16$ & $2 / 4$ & $0.25 / 0.25$ & & Additive/synergistic \\
\hline NIKSA 10 & Oxacillin & $128 / 512$ & $64 / 64$ & $0.5 / 0.125$ & $0.12 / 0.3 / 2$ & \\
\hline
\end{tabular}

${ }^{1}$ The MIC and MBC of cryptotanshinone with oxacillin.

${ }^{2}$ The FIC index.

${ }^{3}$ MSSA (ATCC 25923): reference strain methicillin-sensitive Staphylococcus aureus.

${ }^{4}$ MRSA (ATCC 33591): reference strain methicillin-resistant Staphylococcus aureus.

${ }^{5}$ VRSA 3A063: vancomycin-resistant Staphylococcus aureus isolated clinically.

${ }^{6}$ VRSA 3A066: vancomycin-resistant Staphylococcus aureus isolated clinically.

${ }^{7}$ MSSA (1-2): methicillin-sensitive Staphylococcus aureus isolated clinically.

${ }^{8}$ MRSA (1-16): methicillin-resistant Staphylococcus aureus isolated clinically. 
TABLE 3: Synergistic effects of cryptotanshinone with ampicillin in isolated MRSA, VRSA, MSSA, and some of reference bacteria.

\begin{tabular}{|c|c|c|c|c|c|c|}
\hline \multirow{2}{*}{ Samples } & \multirow{2}{*}{ Agent } & \multicolumn{2}{|c|}{$\mathrm{MIC} / \mathrm{MBC}(\mu \mathrm{g} / \mathrm{mL})$} & \multirow{2}{*}{ FIC/FBC } & \multirow{2}{*}{$\mathrm{FICI} / \mathrm{FBCI}^{2}$} & \multirow{2}{*}{ Outcome } \\
\hline & & Alone & Combination $^{1}$ & & & \\
\hline \multirow{2}{*}{ MSSA ATCC $25923^{3}$} & Cryptotanshinone & $64 / 256$ & $16 / 64$ & $0.25 / 0.25$ & \multirow{2}{*}{$0.5 / 0.75$} & \multirow{2}{*}{ Synergistic/additive } \\
\hline & Ampicillin & $8 / 16$ & $2 / 8$ & $0.25 / 0.5$ & & \\
\hline \multirow{2}{*}{ MRSA ATCC $33591^{4}$} & Cryptotanshinone & $4 / 16$ & $1 / 4$ & $0.25 / 0.25$ & \multirow{2}{*}{$0.5 / 0.5$} & \multirow{2}{*}{ Synergistic/synergistic } \\
\hline & Ampicillin & $512 / 2048$ & $128 / 512$ & $0.25 / 0.25$ & & \\
\hline \multirow{2}{*}{ VRSA $3 \mathrm{~A} 063^{5}$} & Cryptotanshinone & $2 / 4$ & $0.5 / 2$ & $0.25 / 0.5$ & \multirow{2}{*}{$0.5 / 0.375$} & \multirow{2}{*}{ Synergistic/synergistic } \\
\hline & Ampicillin & $512 / 2048$ & $128 / 256$ & $0.25 / 0.125$ & & \\
\hline \multirow{2}{*}{ VRSA $3 \mathrm{~A} 066^{6}$} & Cryptotanshinone & $4 / 16$ & $1 / 4$ & $0.25 / 0.25$ & \multirow{2}{*}{$0.5 / 0.5$} & \multirow{2}{*}{ Synergistic/synergistic } \\
\hline & Ampicillin & $128 / 256$ & $32 / 64$ & $0.25 / 0.25$ & & \\
\hline \multirow{2}{*}{ MSSA $1^{7}$} & Cryptotanshinone & $16 / 64$ & $4 / 8$ & $0.25 / 0.125$ & \multirow{2}{*}{$0.5 / 0.375$} & \multirow{2}{*}{ Synergistic/synergistic } \\
\hline & Ampicillin & $64 / 128$ & $16 / 32$ & $0.25 / 0.25$ & & \\
\hline \multirow{2}{*}{ MSSA 2} & Cryptotanshinone & $32 / 128$ & $8 / 16$ & $0.25 / 0.125$ & \multirow{2}{*}{$0.5 / 0.375$} & Synergistic/synergistic \\
\hline & Ampicillin & $256 / 512$ & $64 / 128$ & $0.25 / 0.25$ & & \\
\hline MRSA $1^{8}$ & Cryptotanshinone & $16 / 32$ & $4 / 8$ & $0.25 / 0.25$ & $0.5 / 0.5$ & Synergistic/synergistic \\
\hline NIKSA 1 & Ampicillin & $128 / 256$ & $32 / 64$ & $0.25 / 0.25$ & $0.3 / 0.3$ & 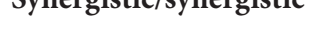 \\
\hline MRSA 2 & Cryptotanshinone & $64 / 128$ & $16 / 32$ & $0.25 / 0.25$ & $0.5 / 05$ & \\
\hline 1VINDA 2 & Ampicillin & $64 / 256$ & $16 / 64$ & $0.25 / 0.25$ & $0.5 / 0.5$ & Synergistic/synergistic \\
\hline MRSA 3 & Cryptotanshinone & $4 / 16$ & $1 / 2$ & $0.25 / 0.125$ & $0.5 / 0.25$ & Synergistic/synergistic \\
\hline & Ampicillin & $128 / 512$ & $32 / 64$ & $0.25 / 0.125$ & $0.5 / 0.23$ & 作 \\
\hline MRSA 4 & Cryptotanshinone & $4 / 8$ & $1 / 2$ & $0.25 / 0.25$ & $0.5 / 0.5$ & Synergistic/synergistic \\
\hline & Ampicillin & $128 / 256$ & $32 / 64$ & $0.25 / 0.25$ & & \\
\hline MRSA 5 & Cryptotanshinone & $64 / 128$ & $16 / 32$ & $0.25 / 0.25$ & $0.5 / 0.5$ & Synergistic/synergistic \\
\hline МाКОА & Ampicillin & $256 / 512$ & $64 / 128$ & $0.25 / 0.25$ & $0.3 / 0.3$ & synergistic/synergistic \\
\hline MRSA 6 & Cryptotanshinone & $4 / 16$ & $1 / 2$ & $0.25 / 0.125$ & $075 / 0625$ & Additive/additive \\
\hline IVIKSA O & Ampicillin & $64 / 128$ & $32 / 64$ & $0.5 / 0.5$ & $0.73 / 0.025$ & 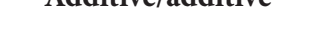 \\
\hline MRSA 7 & Cryptotanshinone & $8 / 16$ & $2 / 4$ & $0.25 / 0.25$ & $0.5 / 0.5$ & Synergistic/synergistic \\
\hline & Ampicillin & $128 / 256$ & $32 / 64$ & $0.25 / 0.25$ & & \\
\hline MRSA 8 & Cryptotanshinone & $32 / 64$ & $8 / 16$ & $0.25 / 0.25$ & $0.5 / 0.375$ & Synergistic/synergistic \\
\hline & Ampicillin & $32 / 64$ & $8 / 8$ & $0.25 / 0.125$ & & \\
\hline MRSA 9 & Cryptotanshinone & $8 / 16$ & $2 / 4$ & $0.25 / 0.25$ & & \\
\hline IVКОА & Ampicillin & $64 / 128$ & $16 / 32$ & $0.25 / 0.25$ & $0.3 / 0.3$ & synergistic/synergistic \\
\hline MRSA 10 & Cryptotanshinone & $16 / 64$ & $2 / 8$ & $0.125 / 0.125$ & $0.375 / 0.375$ & Synergistic/synergistic \\
\hline & Ampicillin & $128 / 256$ & $32 / 64$ & $0.25 / 0.25$ & & \\
\hline MRSA 11 & Cryptotanshinone & $8 / 32$ & $2 / 8$ & $0.25 / 0.25$ & $0.5 / 0.75$ & Synergistic/additive \\
\hline & Ampicillin & $64 / 128$ & $16 / 64$ & $0.25 / 0.5$ & & \\
\hline MRSA 12 & Cryptotanshinone & $4 / 8$ & $1 / 2$ & $0.25 / 0.25$ & $0.75 / 0.5$ & Additive/synergistic \\
\hline VVINOA IL & Ampicillin & $128 / 256$ & $64 / 64$ & $0.5 / 0.25$ & & \\
\hline MRSA 13 & Cryptotanshinone & $32 / 128$ & $8 / 32$ & $0.25 / 0.25$ & $0.5 / 0.75$ & Synergistic/additive \\
\hline IVINUת 1J & Ampicillin & $128 / 256$ & $32 / 128$ & $0.25 / 0.5$ & 0.010 .13 & 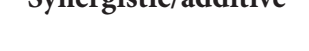 \\
\hline MRSA 14 & Cryptotanshinone & $8 / 16$ & $2 / 4$ & $0.25 / 0.25$ & $0.375 / 0.5$ & Synergistic/synergistic \\
\hline & Ampicillin & $64 / 128$ & $8 / 32$ & $0.125 / 0.25$ & 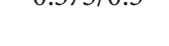 & 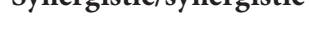 \\
\hline MRSA 15 & Cryptotanshinone & $4 / 16$ & $2 / 4$ & $0.5 / 0.25$ & $0.75 / 0.5$ & Additive/synergistic \\
\hline 1VITU & Ampicillin & $64 / 128$ & $16 / 32$ & $0.25 / 0.25$ & 0.1370 .0 & 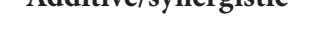 \\
\hline MRSA & Cryptotanshinone & $8 / 16$ & $2 / 4$ & $0.25 / 0.25$ & $0.5 / 0.375$ & Synergistic/synergistic \\
\hline & Ampicillin & $128 / 512$ & $32 / 64$ & $0.25 / 0.125$ & & \\
\hline
\end{tabular}

${ }^{1}$ The MIC and MBC of cryptotanshinone with ampicillin.

${ }^{2}$ The FIC index.

${ }^{3}$ MSSA (ATCC 25923): reference strain methicillin-sensitive Staphylococcus aureus.

${ }^{4}$ MRSA (ATCC 33591): reference strain methicillin-resistant Staphylococcus aureus.

${ }^{5}$ VRSA 3A063: vancomycin-resistant Staphylococcus aureus isolated clinically.

${ }^{6}$ VRSA 3A066: vancomycin-resistant Staphylococcus aureus isolated clinically.

${ }^{7}$ MSSA (1-2): methicillin-sensitive Staphylococcus aureus isolated clinically.

${ }^{8}$ MRSA (1-16): methicillin-resistant Staphylococcus aureus isolated clinically. 
TABLE 4: Synergistic effects of cryptotanshinone with vancomycin in isolated MRSA, VRSA, MSSA, and some of reference bacteria.

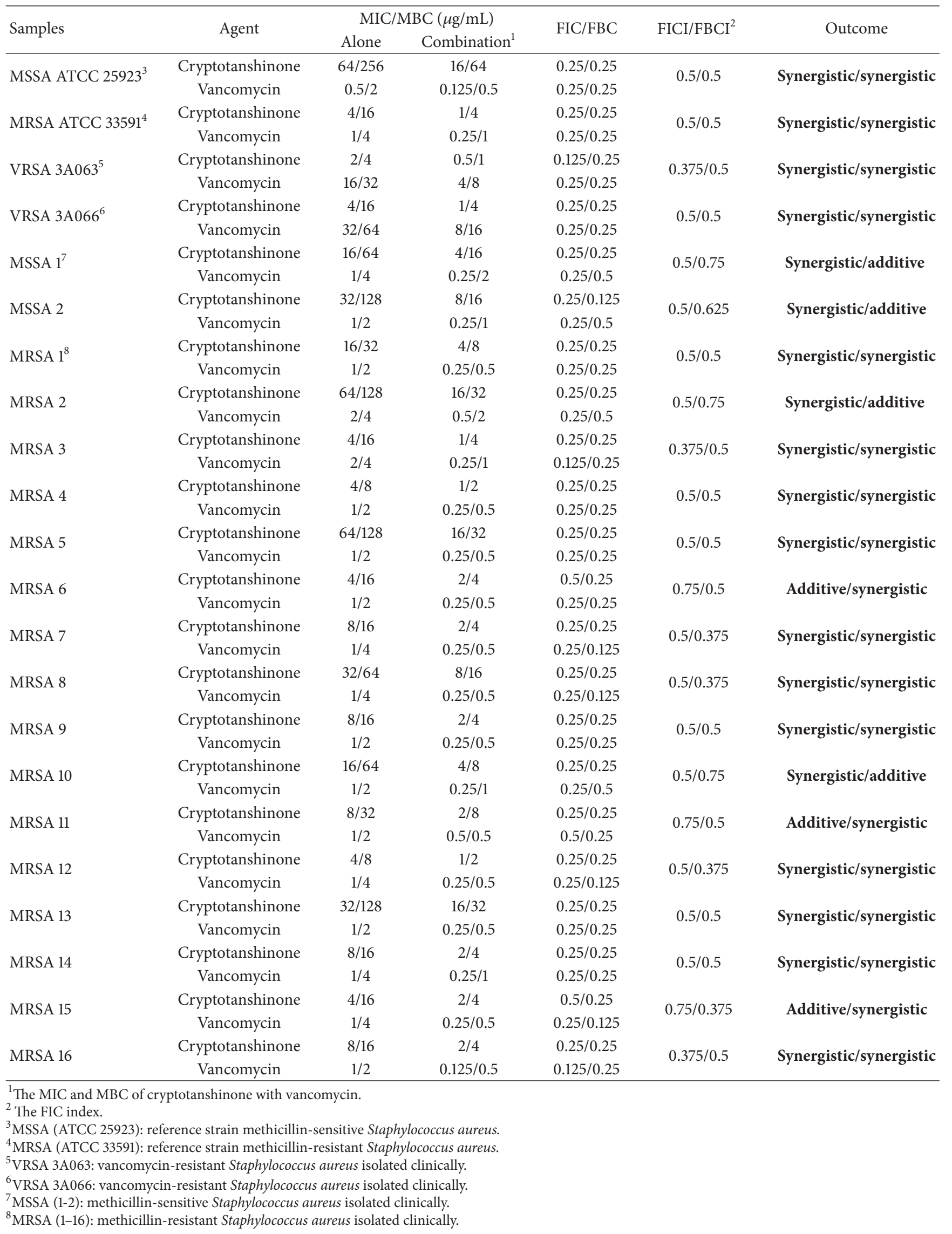


2.2. Preparation of Bacterial Strains. 16 isolates of methicillinresistant Staphylococcus aureus, 2 isolates of methicillinsensitive $S$. aureus (MSSA), and 2 isolates of vancomycinresistant $S$. aureus (VRSA) were purchased from the Culture Collection of Antimicrobial Resistant Microbes (CCARM); standard strains of methicillin-sensitive S. aureus (MSSA) ATCC 25923 and methicillin-resistant S. aureus (MRSA) ATCC 33591 were used as well (Table 1). Antibiotic susceptibility was determined in testing the inhibition zones (inoculums $0.5 \mathrm{McF}$ arland suspension, $1.5 \times 10^{8} \mathrm{CFU} / \mathrm{mL}$ ) and $\mathrm{MIC} / \mathrm{MBC}$ (inoculums $5 \times 10^{5} \mathrm{CFU} / \mathrm{mL}$ ) for strains, measured as described in the National Committee for Clinical Laboratory Standards (NCCLS, 1999). Briefly, the growth of bacteria was examined at $37^{\circ} \mathrm{C}$ in $0.95 \mathrm{~mL}$ of $\mathrm{BHI}$ broth containing various concentrations of CT. These tubes were inoculated with $5 \times 10^{5}$ colony-forming units $(\mathrm{CFU}) / \mathrm{mL}$ of an overnight culture grown in $\mathrm{BHI}$ broth and incubated at $37^{\circ} \mathrm{C}$. After $24 \mathrm{~h}$ of incubation, the optical density (OD) was measured spectrophotometrically at $550 \mathrm{~nm}$. Three replicates were measured for each concentration of tested drugs.

2.3. Minimum Inhibitory Concentration/Minimum Bactericidal Concentration Assay. The antimicrobial activities of CT against clinical isolates MRSA 16, MSSA 2, VRSA 2, and reference strains were determined via the broth dilution method [25]. The minimum inhibitory concentration (MIC) was recorded as the lowest concentration of test samples resulting in the complete inhibition of visible growth. For clinical strains, $\mathrm{MIC}_{50} \mathrm{~s}$ and $\mathrm{MIC}_{90} \mathrm{~s}$, defined as MICs at which 50 and $90 \%$, respectively, of the isolates were inhibited, were determined. The minimum bactericidal concentration (MBC) was determined based on the lowest concentration of the extracts required to kill $99.9 \%$ of bacteria from the initial inoculum as determined by plating on agar.

2.4. Checkerboard Dilution Test. The synergistic combinations were investigated in the preliminary checkerboard method performed using the MRSA, MSSA, and VRSA of clinical isolate strains via MIC and MBC determination [26]. The fractional inhibitory concentration index (FICI) and fractional bactericidal concentration index (FBCI) are the sum of the FICs and FBCs of each of the drugs, which were defined as the MIC and MBC of each drug when used in combination divided by the MIC and MBC of each drug when used alone. The FIC and FBC index was calculated as follows: $\mathrm{FIC}=(\mathrm{MIC}$ of drug $\mathrm{A}$ in combination/MIC of drug A alone $)$ + (MIC of drug B in combination/MIC of drug B alone) and $\mathrm{FBC}=(\mathrm{MBC}$ of drug $\mathrm{A}$ in combination/MBC of drug $\mathrm{A}$ alone $)+(\mathrm{MBC}$ of drug $\mathrm{B}$ in combination/MBC of drug $\mathrm{B}$ alone). FIC and FBC indices were interpreted as follows: the FIC index was interpreted as follows: synergy, <0.5; partial synergy, 0.5-0.75; additive effect, 0.76-1.0; indifference, $>1.0-$ 4.0; and antagonism, $>4.0[26]$.

2.5. Time-Kill Curves. The bactericidal activities of the drugs evaluated in this study were also evaluated using time-kill curves constructed using the isolated and reference strains. Cultures with an initial cell density of $1-5 \times 10^{6} \mathrm{CFU} / \mathrm{mL}$ were exposed to the MIC of CT alone or CT (1/2 MIC) plus oxacillin or ampicillin or vancomycin (1/2 MIC). Viable counts were conducted at $0,0.5,1,2,3,4,5,6,12$, and $24 \mathrm{~h}$ by plating aliquots of the samples on agar and subsequent incubation for 24 hours at $37^{\circ} \mathrm{C}$. All experiments were repeated several times and colony counts were conducted in duplicate, after which the means were determined.

\section{Results and Discussion}

Many researchers are studying natural products that could be used as antibiotics against MRSA and are employing novel dosing regimens and antimicrobials that would be advantageous for combating the therapeutic problems associated with $S$. aureus $[10,13,14,16,27]$. The main bioactive constituents of $S$. miltiorrhiza include water-soluble phenolic acids and lipophilic diterpenoid tanshinones [28-30]. Cryptotanshinone was isolated from dried S. miltiorrhiza roots and identified via comparison of their spectral data with the data reported in the literature $[23,24] .{ }^{1} \mathrm{H}-\mathrm{NMR}$ $\left(\mathrm{CDCl}_{3}\right) \delta 7.64(1 \mathrm{H}, \mathrm{d}, J=8.0 \mathrm{~Hz}), 7.48(1 \mathrm{H}, \mathrm{d}, J=8.0 \mathrm{~Hz})$, $4.86(1 \mathrm{H}, \mathrm{t}, J=9.2 \mathrm{~Hz}), 4.36(1 \mathrm{H}, \mathrm{dd}, J=6.0$ and $6.0 \mathrm{~Hz})$, $3.60(1 \mathrm{H}, \mathrm{m}), 3.21(2 \mathrm{H}$, br t), $1.69(4 \mathrm{H}, \mathrm{m}), 1.36(3 \mathrm{H}, \mathrm{d}, J$ $=6.4 \mathrm{~Hz}), 1.31(6 \mathrm{H}, \mathrm{s}) .{ }^{13} \mathrm{C}-\mathrm{NMR}\left(\mathrm{CDCl}_{3}\right) \delta 29.67(\mathrm{C}-1)$, 19.08 (C-2), 37.82 (C-3), 34.86 (C-4), 143.70 (C-5), 132.56 (C-6), 122.50 (C-7), 128.42 (C-8), 126.27 (C-9), 152.37 (C10), 184.27 (C-11), 175.72 (C-12), 118.30 (C-13), 170.75 (C-14), 81.46 (C-15), 34.62 (C-16), 18.85 (C-17), 31.94 (C-18), and 31.89 (C-19). Among the lipophilic diterpenoid tanshinones, cryptotanshinone, dihydrotanshinone I, tanshinone IIA, and tanshinone I exhibited strong antimicrobial activity against ( Agrobacterium tumefaciens ATCC 11158, Escherichia coli ATCC 29425, Pseudomonas lachrymans ATCC 11921, Ralstonia solanacearum ATCC 11696, and Xanthomonas vesicatoria ATCC 11633) and three Gram-positive bacteria (Bacillus subtilis ATCC 11562, Staphylococcus aureus ATCC 6538, and Staphylococcus haemolyticus ATCC 29970) [18]. Our results of the antibacterial activity showed that the CT exhibited inhibitory activities against isolates MSSA, MRSA, VRSA, and reference stains. The MICs and MBCs values of CT and antibiotics, ampicillin, oxacillin, and vancomycin against MSSA ATCC 25923 and MRSA ATCC 33591 and isolates MSSA 1-2, MRSA 1-16, and VRSA 1-2 are shown in Table 1. The MICs and MBCs values of CT against isolates MSSA $1-2$ were in the range of 16 and $32 \mu \mathrm{g} / \mathrm{mL}$ and 64 and $128 \mu \mathrm{g} / \mathrm{mL}$, isolates MRSA $1-16$ in the range of $4-64 \mu \mathrm{g} / \mathrm{mL}$ and $8-128 \mu \mathrm{g} / \mathrm{mL}$, isolates VRSA $1-2$ in the range of 2 and $4 \mu \mathrm{g} / \mathrm{mL}$ and 4 and $16 \mu \mathrm{g} / \mathrm{mL}$, and reference stains in range of 4 and $64 \mu \mathrm{g} / \mathrm{mL}$ and 16 and $256 \mu \mathrm{g} / \mathrm{mL}$, respectively. The MICs/MBCs for ampicillin were determined to be either $8 / 16$ or $1024 / 2048 \mu \mathrm{g} / \mathrm{mL}$; for oxacillin, either $0.25 / 1$ or $512 / 2048 \mu \mathrm{g} / \mathrm{mL}$; for vancomycin, either $0.5 / 2$ or $32 / 64 \mu \mathrm{g} / \mathrm{mL}$ against reference strains and MSSA 1-2, MRSA 1-16, and VRSA 1-2 isolates. The $\mathrm{MIC}_{50}$ and $\mathrm{MIC}_{90}$ values of CT for reference strains were 0.5 and $4 \mu \mathrm{g} / \mathrm{mL}$ and 4 and $64 \mu \mathrm{g} / \mathrm{mL}$, while for MSSA 1-2 and VRSA 1-2 isolates were $0.5-8 \mu \mathrm{g} / \mathrm{mL}$ and $2-32 \mu \mathrm{g} / \mathrm{mL}$, and for MRSA 1-16 isolates were 0.5$8 \mu \mathrm{g} / \mathrm{mL}$ and $4-64 \mu \mathrm{g} / \mathrm{mL}$, respectively (Table 1 ). 

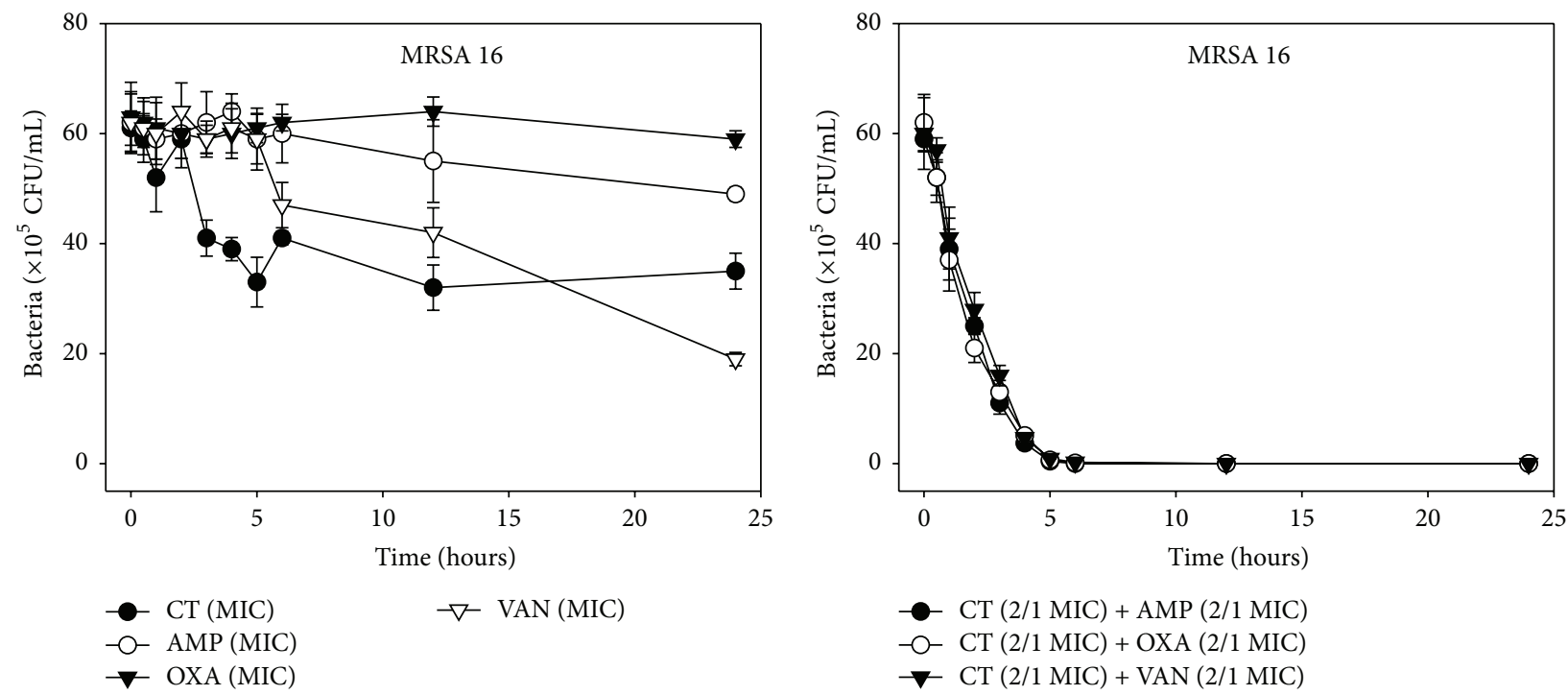

FIGURE 8: Time-kill curves of MIC or 1/2 MIC of cryptotanshinone (CT), ampicillin (AMP), oxacillin (OXA), and vancomycin (VAN) alone and its combination with 1/2 MIC of AMP or OXA, and VAN against MRSA 16 isolate. Bacteria were incubated with MIC of CT (๑), AMP $(\bigcirc)$, OXA $(\boldsymbol{\nabla})$, and VAN $(\nabla)$, and 1/2 MIC of CT + 1/2 MIC of AMP $(\bullet), 1 / 2 \mathrm{MIC}$ of CT + 1/2 MIC of OXA (O), and 1/2 MIC of CT + 1/2 MIC of VAN $(\boldsymbol{\nabla})$ over time. CFU: colony-forming units.

Evaluation of in vivo effectiveness of the antimicrobial combinations is necessary to generate data that can be extrapolated to the clinical situation as well as predicting relevant concentration of optimal dosing regimens for both agents of the combinations [31,32]. Combination antibiotic therapy has been studied to promote the effective use of antibiotics in increasing in vivo activity of antibiotics, in preventing the spread of drug-resistant strains, and in minimizing toxicity $[32,33]$. The combination of oxacillin and CT resulted in a reduction in the MICs/MBCs for isolates VRSA 1-2 and MSSA $1-2$, with the MICs/MBCs of $2 / 16$ or $8 / 128 \mu \mathrm{g} / \mathrm{mL}$, for oxacillin becoming $0.125 / 0.125-0.25 \mu \mathrm{g} / \mathrm{mL}$ and reduced by $\geq 4$-fold, evidencing a synergistic effect as defined by a FICI and FBCI of $\leq 0.5$ (Table 2). The combination of oxacillin and CT resulted in a reduction against isolates MRSA 116 , with the MICs/MBCs values of $0.5-16 / 2-32 \mu \mathrm{g} / \mathrm{mL}$, for oxacillin becoming $1-128 / 4-256 \mu \mathrm{g} / \mathrm{mL}$ and reduced by $\geq 4$ fold, evidencing a synergistic effect as defined by a FICI and FBCI of $\leq 0.5$ except MRSA 4 and 16 of additive (FICI $\geq$ 0.5 ) and MRSA 1, 7, and 15 of additive (FBCI $\geq 0.5$ ). The combination of ampicillin and CT resulted in a reduction in the MICs/MBCs for isolates VRSA 1-2 and MSSA 12 , with the MICs/MBCs of $0.5 / 1$ or $2 / 4 \mu \mathrm{g} / \mathrm{mL}$ and $4 / 8$ or $8 / 16$, for ampicillin becoming $32 / 128$ or $64 / 256 \mu \mathrm{g} / \mathrm{mL}$ and $16 / 64$ or $32 / 128 \mu \mathrm{g} / \mathrm{mL}$ and reduced by $\geq 4$-fold, evidencing a synergistic effect as defined by a FICI and FBCI of $\leq 0.5$ and in most of MRSA tested were reduced by $\geq 4$-fold evidencing a synergistic effect as defined by a FICI and FBCI of $\leq 0.5$ except MRSA 6, 12, and 15 (FICI $\geq 0.5$ ) and MRSA 6, 11, and 13 (FBCI $\geq 0.5$ ), respectively (Table 3 ). The combination of vancomycin and CT resulted in a reduction against isolates MRSA 116 , with the MICs/MBCs values of $1-16 / 2-32 \mu \mathrm{g} / \mathrm{mL}$, for vancomycin becoming $0.125-0.5 / 0.5-2 \mu \mathrm{g} / \mathrm{mL}$ and reduced by $\geq 4$-fold, evidencing a synergistic effect as defined by a FICI and FBCI of $\leq 0.5$ except MRSA 6, 11 , and 15 of additive (FICI $\geq 0.5$ ) and MRSA 1 and 9 of additive (FBCI $\geq 0.5$ ) and for isolates VRSA 1-2 and MSSA 1-2, with the MICs/MBCs of 0.5/1 or $1 / 4 \mu \mathrm{g} / \mathrm{mL}$ and $4 / 8$ or $16 / 16$, for vancomycin becoming $4 / 8$ or $8 / 16 \mu \mathrm{g} / \mathrm{mL}$ and $0.25 / 2$ or $0.25 / 1 \mu \mathrm{g} / \mathrm{mL}$ and reduced by $\geq 4$ fold, evidencing a synergistic effect as defined by a FICI and FBCI of $\leq 0.5$ except MSSA 1 and 2 of additive (FBCI $\geq 0.5$ ) (Table 4).

The effects of CT administered in combination with oxacillin and/or ampicillin and/or vancomycin against standard (MSSA and MRSA) and clinical isolates of MSSA (1, 2), VRSA (1,2), and MRSA (MRSA 1-16) were confirmed by time-kill curve experiments (Figures 1, 2, 3, 4, 5, 6, 7 , and 8). Cultures of each strain of bacteria with a cell density of $10^{6} \mathrm{CFU} / \mathrm{mL}$ were exposed to the MIC of CT and antibiotics alone or CT (1/2 MIC) with oxacillin (1/2 MIC), ampicillin (1/2 MIC), or vancomycin (1/2 MIC). We observed that 30 minutes of CT treatment with ampicillin, oxacillin, or vancomycin resulted in an increased rate of killing as compared to that observed with $\mathrm{CT}$ (MIC) alone. A profound bactericidal effect was exerted when a combination of drugs was utilized. The growth of the tested bacteria was completely attenuated after $2-5 h$ of treatment with the $1 / 2$ MIC of CT, regardless of whether it was administered alone or with oxacillin (1/2 MIC), ampicillin (1/2 MIC), or vancomycin (1/2 MIC) (Figures 1-8).

It has been reported that some plant-derived compounds can improve the in vitro activity of some cell-wall inhibiting antibiotics by directly attacking the same target site, that is, peptidoglycan $[7,9,34]$. CT exhibits antimicrobial 
activity against a broad range of Gram-positive bacteria, including $S$. aureus, and Gram-negative bacteria as well as other microorganisms [21, 22]. Despite the pharmacological activities, potential risks regarding combination use of S. miltiorrhiza and drugs have been observed [28, 35, 36]. Scientific findings concluded $S$. miltiorrhiza fraction, and its components (cryptotanshinone and dihydrotanshinone I) showed antibacterial activity against a broad range of bacteria, including the broad range of Gram-positive bacteria and Gram-negative bacteria, and superoxide radicals are considered important in the antibacterial actions of the agents [18]. The Gram-positive bacteria-specific properties of CT are caused by the inhibition of RNA and protein synthesis, rather than by attacking the bacterial membrane [21, 22].

In conclusion, CT of $S$. miltiorrhiza is expected to be recognized as natural sources for the development of new functional drugs against multiresistant $S$. aureus, MRSA and VRSA.

\section{Conflict of Interests}

The authors have declared no conflict of interests.

\section{Authors' Contribution}

Jeong-Dan Cha and Jeong-Ho Lee contributed equally to this work.

\section{Acknowledgment}

This research was supported by Dong-eui University Grant (2011AA106).

\section{References}

[1] G. R. Corey, "Staphylococcus aureusbloodstream infections: definitions and treatment," Clinical Infectious Diseases, vol. 48, no. 4, pp. S254-S259, 2009.

[2] C. A. Petti and V. G. Fowler Jr., "Staphylococcus aureus bacteremia and endocarditis," Cardiology Clinics, vol. 21, no. 2, pp. 219-233, 2003.

[3] A. Malm, A. Biernasiuk, R. Łoś et al., "Slime production and cell surface hydrophobicity of nasopharyngeal and skin staphylococci isolated from healthy people," Polish Journal of Microbiology, vol. 54, no. 2, pp. 117-121, 2005.

[4] S. Defres, C. Marwick, and D. Nathwani, "MRSA as a cause of lung infection including airway infection, community-acquired pneumonia and hospital-acquired pneumonia," European Respiratory Journal, vol. 34, no. 6, pp. 1470-1476, 2009.

[5] D. P. Levine, "Vancomycin: understanding its past and preserving its future," Southern Medical Journal, vol. 101, no. 3, pp. 284291, 2008.

[6] Z. Moravvej, F. Estaji, E. Askari, K. Solhjou, M. N. Nasab, and S. Saadat, "Update on the global number of vancomycin-resistant Staphylococcus aureus (VRSA) strains," International Journal of Antimicrobial Agents, vol. 42, no. 4, pp. 370-371, 2013.
[7] C.-H. Huang and Y.-H. Chen, "The detection and clinical impact of vancomycin MIC among patients with methicillinresistant Staphylococcus aureus bacteremia," Journal of Microbiology, Immunology and Infection, vol. 46, no. 4, pp. 315-316, 2013.

[8] D. I. Hsu, L. K. Hidayat, R. Quist et al., "Comparison of methodspecific vancomycin minimum inhibitory concentration values and their predictability for treatment outcome of meticillinresistant Staphylococcus aureus (MRSA) infections," International Journal of Antimicrobial Agents, vol. 32, no. 5, pp. 378-385, 2008.

[9] H. C. Maltezou and H. Giamarellou, "Community-acquired methicillin-resistant Staphylococcus aureus infections," International Journal of Antimicrobial Agents, vol. 27, no. 2, pp. 87-96, 2006.

[10] F. Aqil, M. S. A. Khan, M. Owais, and I. Ahmad, "Effect of certain bioactive plant extracts on clinical isolates of $\beta$-lactamase producing methicillin resistant Staphylococcus aureus," Journal of Basic Microbiology, vol. 45, no. 2, pp. 106-114, 2005.

[11] J. N. Eloff, "Which extractant should be used for the screening and isolation of antimicrobial components from plants?" Journal of Ethnopharmacology, vol. 60, no. 1, pp. 1-8, 1998.

[12] J. Parekh and S. V. Chanda, "In vitro antimicrobial activity and phytochemical analysis of some Indian medicinal plants," Turkish Journal of Biology, vol. 31, no. 1, pp. 53-58, 2007.

[13] T. Hatano, Y. Shintani, Y. Aga, S. Shiota, T. Tsuchiya, and T. Yoshida, "Phenolic constituents of licorice. VIII. Structures of glicophenone and glicoisoflavanone, and effects of licorice phenolics on methicillin-resistant Staphylococcus aureus," Chemical and Pharmaceutical Bulletin, vol. 48, no. 9, pp. 1286-1292, 2000.

[14] T. Fukai, A. Marumo, K. Kaitou, T. Kanda, S. Terada, and T. Nomura, "Antimicrobial activity of licorice flavonoids against methicillin-resistant Staphylococcus aureus," Fitoterapia, vol. 73, no. 6, pp. 536-539, 2002.

[15] E. Tripoli, M. L. Guardia, S. Giammanco, D. D. Majo, and M. Giammanco, "Citrus flavonoids: molecular structure, biological activity and nutritional properties: a review," Food Chemistry, vol. 104, no. 2, pp. 466-479, 2007.

[16] G. B. Mahady, "Medicinal plants for the prevention and treatment of bacterial infections," Current Pharmaceutical Design, vol. 11, no. 19, pp. 2405-2427, 2005.

[17] J. Cao, Y.-J. Wei, L.-W. Qi et al., "Determination of fifteen bioactive components in Radix et Rhizoma Salviae Miltiorrhizae by high-performance liquid chromatography with ultraviolet and mass spectrometric detection," Biomedical Chromatography, vol. 22, no. 2, pp. 164-172, 2008.

[18] D.-S. Lee, S.-H. Lee, J.-G. Noh, and S.-D. Hong, "Antibacterial activities of cryptotanshinone and dihydrotanshinone I from a medicinal herb, Salvia miltiorrhiza bunge," Bioscience, Biotechnology and Biochemistry, vol. 63, no. 12, pp. 2236-2239, 1999.

[19] Y.-M. Ahn, S. K. Kim, S.-H. Lee et al., "Renoprotective effect of Tanshinone IIA, an active component of Salvia miltiorrhiza, on rats with chronic kidney disease," Phytotherapy Research, vol. 24, no. 12, pp. 1886-1892, 2010.

[20] W. Chen, L. Liu, Y. Luo et al., "Cryptotanshinone activates p38/JNK and inhibits Erk1/2 leading to caspase-independent cell death in tumor cells," Cancer Prevention Research, vol. 5, no. 5, pp. 778-787, 2012.

[21] H. Feng, H. Xiang, J. Zhang et al., "Genome-wide transcriptional profiling of the response of Staphylococcus aureus to 
cryptotanshinone," Journal of Biomedicine and Biotechnology, vol. 2009, Article ID 617509, 8 pages, 2009.

[22] J.-W. Lee, Y.-J. Ji, S.-O. Lee, and I.-S. Lee, "Effect of Saliva miltiorrhiza Bunge on antimicrobial activity and resistant gene regulation against Methicillin-Resistant Staphylococcus aureus (MRSA)," Journal of Microbiology, vol. 45, no. 4, pp. 350-357, 2007.

[23] M. Gu, G. Zhang, Z. Su, and F. Ouyang, "Identification of major active constituents in the fingerprint of Salvia miltiorrhiza Bunge developed by high-speed counter-current chromatography," Journal of Chromatography A, vol. 1041, no. 1-2, pp. 239243, 2004.

[24] E. J. Park, H. Y. Ji, N. J. Kim et al., "Simultaneous determination of tanshinone I, dihydrotanshinone I, tanshinone IIA and cryptotanshinone in rat plasma by liquid chromatographytandem mass spectrometry: application to a pharmacokinetic study of a standardized fraction of Salvia miltiorrhiza, PF2401SF, Biomedical Chromatography, vol. 22, no. 5, pp. 548-555, 2008.

[25] R. D. Wojtyczka, A. Dziedzic, D. Idzik et al., "Susceptibility of Staphylococcus aureus clinical isolates to propolis extract alone or in combination with antimicrobial drugs," Molecules, vol. 18, no. 8, pp. 9623-9640, 2013.

[26] W. Seesom, A. Jaratrungtawee, S. Suksumran, C. Mekseepralard, P. Ratananukul, and W. Sukhumsirichart, "Antileptospiral activity of xanthones from Garcinia mangostana and synergy of gamma-mangostin with penicillin G.," BMC Complementary and Alternative Medicine, vol. 13, article 1, 2013.

[27] A. Ribeiro, A. Z. Coronado, M. C. Silva-Carvalho et al., "Detection and characterization of international communityacquired infections by methicillin-resistant Staphylococcus aureus clones in Rio de Janeiro and Porto Alegre cities causing both community- and hospital-associated diseases," Diagnostic Microbiology and Infectious Disease, vol. 59, no. 3, pp. 339-345, 2007.

[28] D. Yuan, Y.-N. Pan, W.-W. Fu, T. Makino, and Y. Kano, "Quantitative analysis of the marker compounds in Salvia miltiorrihiza root and its phytomedicinal preparations," Chemical and Pharmaceutical Bulletin, vol. 53, no. 5, pp. 508-514, 2005.

[29] Y.-G. Li, L. Song, M. Liu, Z.-B. Zhi-Bi-Hu, and Z.-T. Wang, "Advancement in analysis of Salviae miltiorrhizae Radix et Rhizoma (Danshen)," Journal of Chromatography A, vol. 1216, no. 11, pp. 1941-1953, 2009.

[30] G. Zeng, H. Xiao, J. Liu, and X. Liang, "Identification of phenolic constituents in Radix Salvia miltiorrhizae by liquid chromatography/electrospray ionization mass spectrometry," Rapid Communications in Mass Spectrometry, vol. 20, no. 3, pp. 499-506, 2006.

[31] C. Jacqueline, J. Caillon, V. Le Mabecque et al., "In vitro activity of linezolid alone and in combination with gentamicin, vancomycin or rifampicin against methicillin-resistant Staphylococcus aureus by time-kill curve methods," Journal of Antimicrobial Chemotherapy, vol. 51, no. 4, pp. 857-864, 2003.

[32] O. Aiyegoro, A. Adewusi, S. Oyedemi, D. Akinpelu, and A. Okoh, "Interactions of antibiotics and methanolic crude extracts of Afzelia Africana (Smith.) against drug resistance bacterial isolates," International Journal of Molecular Sciences, vol. 12, no. 7, pp. 4477-4487, 2011.

[33] J.-Y. Lee, S. O. Won, S. K. Kwan et al., "Synergy of arbekacinbased combinations against vancomycin hetero-intermediate
Staphylococcus aureus," Journal of Korean Medical Science, vol. 21, no. 2, pp. 188-192, 2006.

[34] M. N. Alekshun and S. B. Levy, "Molecular mechanisms of antibacterial multidrug resistance," Cell, vol. 128, no. 6, pp. 10371050, 2007.

[35] T.-H. Chen, Y.-T. Hsu, C.-H. Chen, S.-H. Kao, and H.M. Lee, "Tanshinone IIA from Salvia miltiorrhiza induces heme oxygenase-1 expression and inhibits lipopolysaccharideinduced nitric oxide expression in RAW 264.7 cells," Mitochondrion, vol. 7, no. 1-2, pp. 101-105, 2007.

[36] J. D. Adams, R. Wang, J. Yang, and E. J. Lien, "Preclinical and clinical examinations of Salvia miltiorrhiza and its tanshinones in ischemic conditions," Chinese Medicine, vol. 1, article 3, 2006. 


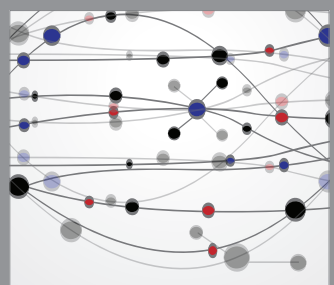

The Scientific World Journal
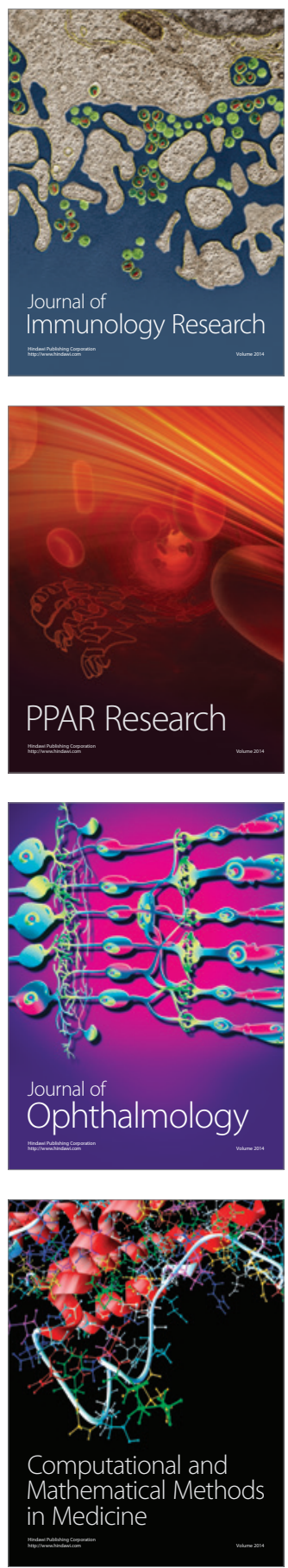

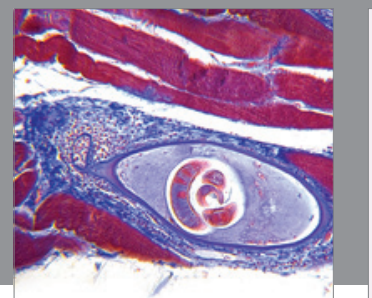

Gastroenterology

Research and Practice
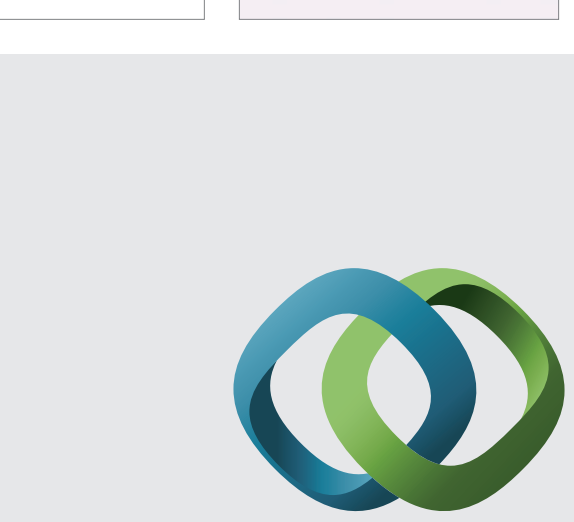

\section{Hindawi}

Submit your manuscripts at

http://www.hindawi.com
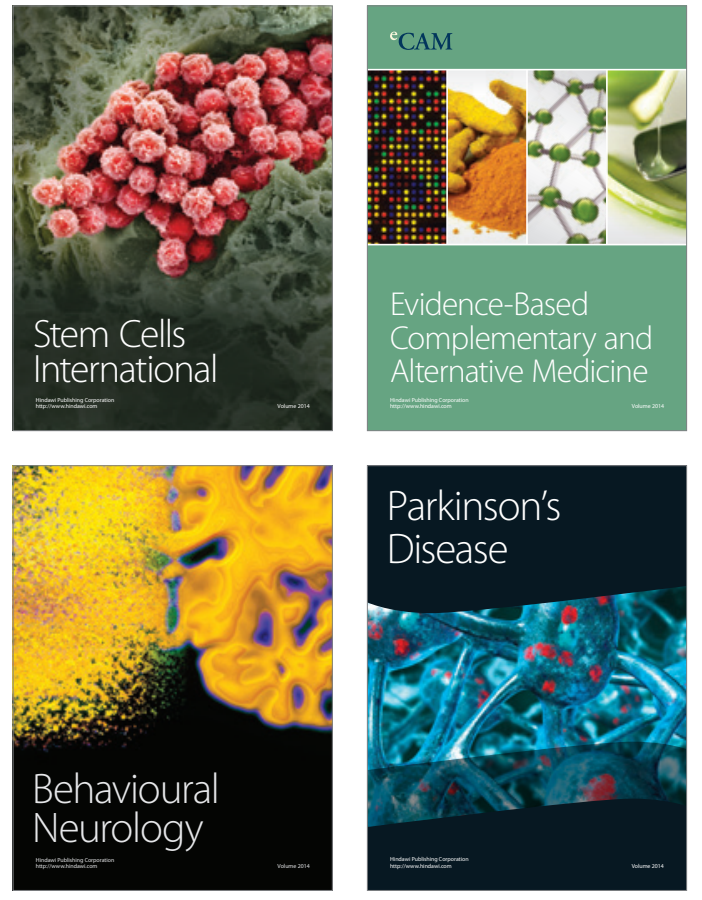
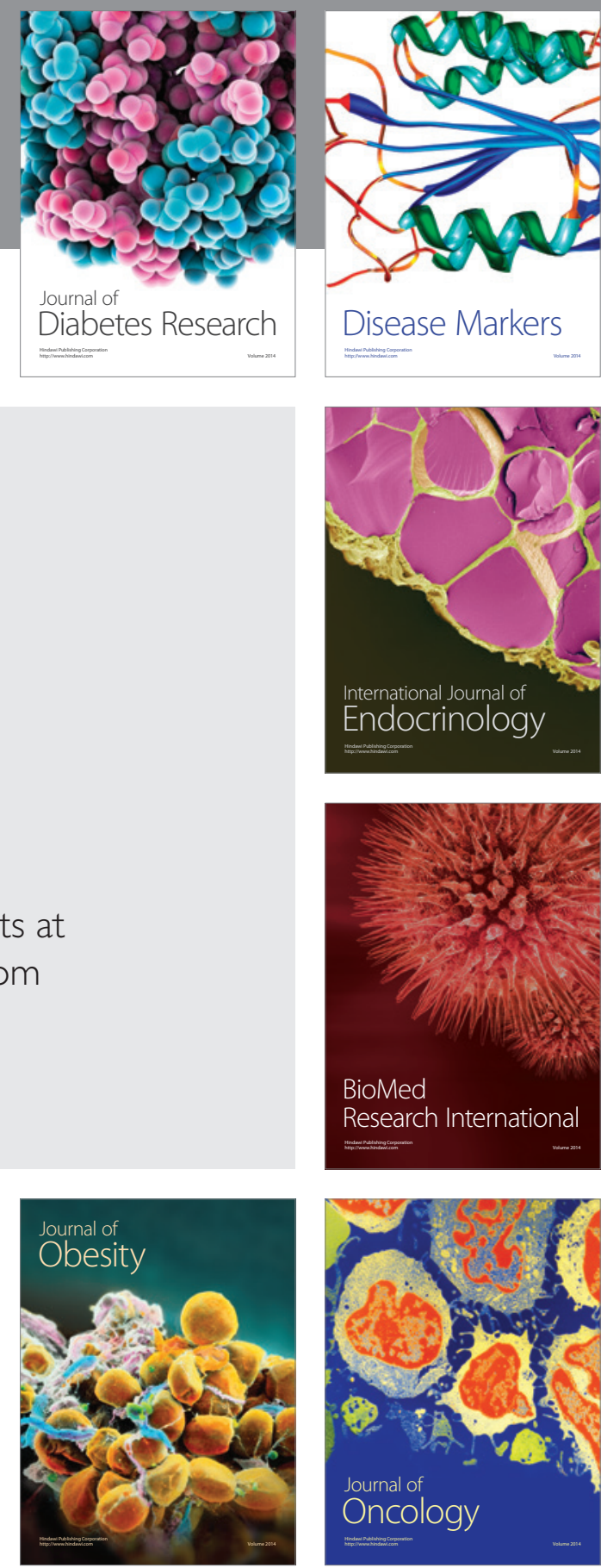

Disease Markers
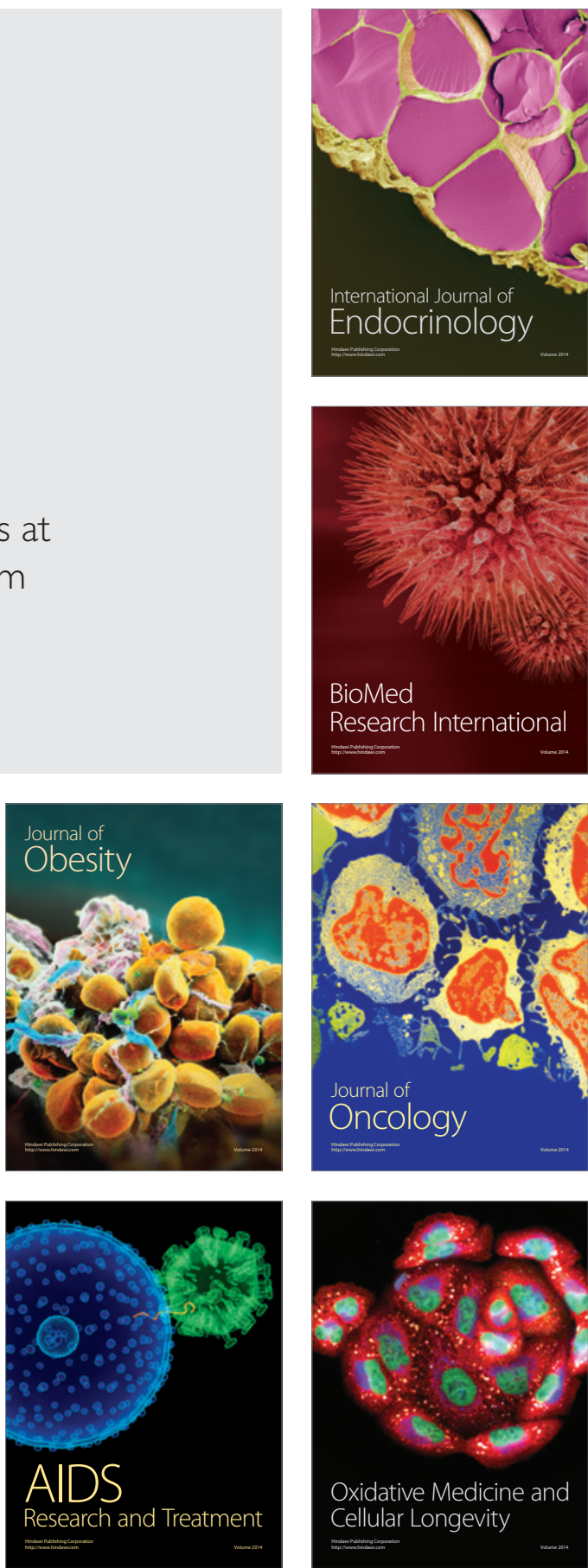